DAMTP-R96/11

\title{
Classical and Quantum Initial Value Problems for Models of Chronology Violation
}

\author{
C.J. Fewster* and A. Higuchi ${ }^{\dagger}$ \\ Institut für theoretische Physik, Universität Bern, Sidlerstrasse 5, CH-3012 Bern, Switzerland \\ and Department of Mathematics, University of York, \\ Heslington, York YO1 5DD, United Kingdom \\ C.G. Wells ${ }^{\S}$ \\ Department of Applied Mathematics and Theoretical Physics, University of Cambridge, \\ Silver St., Cambridge CB3 9EW, United Kingdom
}

(October 24, 2018)

\begin{abstract}
We study the classical and quantum theory of a class of nonlinear differential equations on chronology violating spacetime models in which space consists of finitely many discrete points. Classically, in the linear and weakly nonlinear regimes (for generic choices of parameters) we prove existence and uniqueness of solutions corresponding to initial data specified before the dischronal region; however, uniqueness (but not existence) fails in the strongly coupled regime. The evolution preserves the symplectic structure.

The quantum theory is approached via the quantum initial value problem (QIVP); that is, by seeking operator-valued solutions to the equation of motion with initial data representing the canonical (anti)commutation relations. Using normal operator ordering, we construct solutions to the QIVP for both Bose and Fermi statistics (again for generic choice of parameters) and prove that these solutions are unique. For models with two spatial points, the resulting evolution is unitary; however, for a more general model the evolution fails to preserve the (anti)commutation relations and is therefore nonunitary.
\end{abstract}

\footnotetext{
${ }^{\ddagger}$ Current address of both CJF and $\mathrm{AH}$

*Electronic address: cjf3@unix.york.ac.uk

$\dagger$ Electronic address: ah28@unix.york.ac.uk

§Electronic address: C.G.Wells@damtp.cam.ac.uk
} 
We show that this nonunitary evolution cannot be described using a superscattering operator with the usual properties.

We present numerical evidence to show that the bosonic quantum theory can pick out a unique classical limit for certain ranges of the coupling strength, even when there are many classical solutions. We show that the quantum theory depends strongly on the choice of operator ordering.

In addition, we show that our results differ from those obtained using the "self-consistent path integral". It follows that the path integral evolution does not correspond to a solution of the equation of motion.

03.70.+k, 04.90.+e

Typeset using REVTEX 


\section{INTRODUCTION}

Spacetimes containing closed timelike curves (CTC's) provide an intriguing environment for the formulation of both classical and quantum physics. Because the present is influenced by both the past and the future, neither existence nor uniqueness is guaranteed a priori for solutions to initial value problems for particles and fields on such spacetimes; these issues underlie many of the apparent paradoxes associated with time travel. In this paper, we attempt to gain insight into the initial value problem for a class of nonlinear differential equations (which may be regarded as toy field theories) on "spacetimes" of a type introduced by Politzer [1]. These spacetimes are defined by taking the Cartesian product of a number of discrete points (representing space) with the real line (time) and then imposing certain identifications to introduce CTC's. The simple nature of these models removes many technical problems and allows us to pursue the analysis to its end.

Previous studies of classical initial value problems on chronology violating spacetimes have mostly focussed on linear fields [2] 6] and billiard ball models [4, 7, 9]. Deutsch [10] has also studied examples of classical computational networks with chronology violating components. To the best of our knowledge there have been no studies of nonlinear fields, except insofar as the billiard ball models may be regarded as providing insight into the strongly nonlinear limit. For linear fields, it turns out that one can formulate a well posed initial value problem under certain conditions. Friedman and Morris [2,3] have rigorously proved existence and partial uniqueness results for massless fields propagating on a class of smooth static wormhole spacetimes with data specified at past null infinity. In addition, they have conjectured that the initial value problem is well posed for asymptotically flat spacetimes with a compact nonchronal region whose past and future regions are globally hyperbolic whenever the problem is well posed in the geometric optics limit. It is much easier to prove existence and uniqueness for linear fields on certain non-smooth chronology violating spacetimes [5,6].

In the billiard ball case, Echeverria et al. [7] and (for similar and more elaborate systems) Novikov [8] have shown that the initial value problem is often ill posed in the sense that the evolution is nonunique; moreover, Rama and Sen [9] have given similar examples in which there appears to be no global self-consistent solution for certain initial data.

One would expect that nonlinear fields should interpolate between the behaviour exhibited by linear fields on one hand and billiard balls on the other. In this paper, we will show that this is indeed the case for our class of models: we prove that the initial value problem is well posed for arbitrary data specified before the dischronal region in both the linear and weakly nonlinear case, but uniqueness (though not existence) fails in the strongly nonlinear regime. In addition to these analytic results, we give an explicit example to demonstrate the lack of uniqueness for a particular value of "coupling strength". We also show that the evolution from the past of the dischronal region to its future preserves the symplectic structure.

The loss of uniqueness for interacting systems on chronology violating spacetimes entails that classical physics loses its predictive power. Various authors have expressed the hope that quantum dynamics on such spacetimes might be better behaved than its classical counterpart, with attention focussing on spacetimes possessing both initial and final chronal regions. Friedman et al. [11] considered linear quantum fields and showed that, 
provided the classical initial value problem is well posed, the quantum evolution between spacelike surfaces in the initial and final chronal regions is unitary; a conclusion borne out by Boulware [12] in a Gott space example (see also [1.5.6] for related results). However, the situation is very different for interacting fields. Both Boulware [12] and Friedman et al. [13] found that the $S$-matrix between the initial and final chronal regions fails to be unitary in perturbative $\lambda \phi^{4}$ theory. Politzer also obtained similar perturbative results in quantum mechanics [5] and also some nonperturbative results in exactly soluble models [1] in which nonunitarity also arises. It is also worth pointing out that some interacting systems do have unitary quantum theories [5].

The breakdown of unitarity raises many problems for the probability interpretation of quantum theory; in particular, ambiguities arise in assigning probabilities to the outcomes of measurements conducted before [13] or spacelike separated from [14] the nonchronal region. There have been various reactions to these problems. Firstly, Hartle 15 has discussed how nonunitary evolutions can be accommodated within the framework of generalised quantum mechanics, and a similar proposal has also been advanced by Friedman et al. [13]. A second approach has been to "repair" the theory by modifying the evolution to yield a unitary theory [16,17]. Thirdly, Hawking [18] has argued that one should expect loss of quantum coherence in the presence of CTC's and that the evolution should be specified by means of a superscattering operator (i.e., a linear mapping from initial to final density matrices) which moreover would not factorise into a unitary $S$-matrix and its adjoint. On this view, the quantity computed using the usual rules for the $S$-matrix is not the physically relevant quantity and its nonunitarity is irrelevant. Rather, one should compute the matrix elements of the superscattering operator. Deutsch [10] has also advocated a density matrix formalism in the context of quantum computational networks (see also [1]). However, this prescription turns out to be nonlinear in the initial density matrix [19].

For the most part, the quantisation method employed in discussions of chronology violation has been based on path integrals in which one sums over all consistent trajectories or field configurations. In this paper, we follow an operator approach based on the quantum initial value problem (QIVP). Namely, we seek operator valued solutions to the equation of motion and any consistency conditions arising from the CTC's, with initial data specified before the dischronal region and forming a representation of the canonical (anti)commutation relations. If there exists a unique solution with this initial data, and the evolved data to the future of the dischronal region also represents the commutation relations, then we say that the QIVP is well posed.

We will show that the QIVP is well posed for all linear models in our class of interest with both Bose and Fermi statistics. The corresponding quantum theory is unitary and agrees with that derived by path integral methods. In the interacting case, we prove the remarkable fact that (with normal ordering) the QIVP always has a unique solution and describe how this solution may be constructed. To obtain more specific results, we consider the cases in which "space" consists of either 2 or 3 points. In the 2-point model, we find that the unique solution to the QIVP satisfies the CCR/CAR's to the future of the nonchronal region (so the QIVP is well posed) and that in consequence the resulting quantum theory is unitary. This contrasts strongly with the corresponding path integral result (generalising that of Politzer [1]) in which the evolution is found to be nonunitary. In consequence, and because the path integral also employs normal ordering, we conclude that the self-consistent 
path integral evolution does not generally correspond to a solution of the equation of motion. Given the different starting points of the two approaches this is not entirely surprising.

In the 3-point model, we show that the QIVP is ill posed for both Bose and Fermi statistics because the evolved data does not satisfy the CCR/CAR's to the future of the dischronal region. The corresponding quantum theory is therefore not unitary. We then discuss the nature of this evolution in the fermionic case in order to determine whether or not it can be described by means of a superscattering operator. To do this it is necessary to translate our results from the Heisenberg picture to the Schrödinger picture. Although there is no unique translation prescription (as a consequence of the violation of the CAR's), we are nonetheless able to show that no Schrödinger picture evolution consistent with the QIVP solution can factorise into the product of an operator and its adjoint, lending support to one element of Hawking's position [18]. However, it also transpires that no such Schrödinger picture evolution can be described by a superscattering matrix as it must either increase the trace of density matrices or map them to non-positive operators. In this sense, the loss of unitarity in our model is much more radical than envisaged by Hawking.

We also study the classical limit of our quantum theory. When this limit exists uniformly one recovers exactly one solution to the classical equation of motion. One might imagine that this limit would fail when the classical theory is nonunique; however, this is not the case. It appears that there are bands of "coupling strength" for which the limit does exist even when there are many classical solutions. We present numerical evidence to exemplify this behaviour including an example in which the quantum theory selects precisely one out of 25 classical solutions. We believe the convergence bands continue to appear as the coupling strength is increased indefinitely. Within the convergence bands, our quantum theory resolves the classical nonuniqueness; for other values of the coupling strength, it is arguable that no classical solution is physically relevant.

In addition, we consider the effect of altering the operator ordering used and find that the solutions to the QIVP can become nonunique for large quantum numbers for non-normal operator ordering. We study a 1-parameter family of operator orderings for the 3-point model and show that the resulting quantum theories are all nonunitary.

The paper is structured as follows. We first describe our class of chronology violating models in Section \I and then study the classical initial value problem for both linear and nonlinear fields in Sect. III. Next, in Sect. IV we discuss the quantum initial value problem for our models in the absence of CTC's and demonstrate its equivalence with canonical quantisation. This serves to fix our notation and definitions for Section $\nabla$ in which we uniquely solve the QIVP with CTC's present, and discuss the 2- and 3-point models, showing that the CCR/CAR's are violated in the 3-point case. This nonunitary evolution is investigated in Sect. VI and is shown not to be described by a superscattering operator. Section VII treats the classical limit, whilst Sect. VIII contains a brief discussion of the effect of operator ordering on our results. In Sect. [X], we review the self-consistent path integral formalism, extending and in one instance correcting the treatment given by Politzer [1]. We use this formalism to compute the general (unitary) evolution for the free models, obtaining agreement with the QIVP. For the 2- and 3-point interacting models we show that the QIVP and path integral differ. We comment on this and other issues in the Conclusion (Sect. 囚). There are three Appendices. Appendix A reproduces our treatment of the free classical evolution using the methods of Goldwirth et al. [6], whilst Appendix B gives a derivation 
of the quantum evolution of the free models using the formalism of Politzer [1], rather than the more direct method employed in the text. In Appendix C, we present the details of a calculation which shows that the CCR/CAR's are violated in the 3-point interacting model.

\section{A CLASS OF CHRONOLOGY VIOLATING MODELS}

In this section, we describe a class of nonlinear differential equations on "spacetimes" in which "space" consists of finitely many discrete points. By making identifications in these spacetimes, we introduce CTC's and obtain spacetime models generalising that studied by Politzer [1]. These identifications are implemented in the field theory by imposing certain boundary conditions which place constraints on the theory.

Let $\mathcal{S}$ be a finite collection of points $\mathcal{S}=\left\{z_{\alpha} \mid \alpha=1, \ldots, s\right\}$ for some $s \geq 2$, and define spacetime to be the Cartesian product $\mathcal{S} \times \mathbb{R}$. We define $\mathfrak{H}$ to be the Hilbert space of complex-valued functions on $\mathcal{S}$ with inner product $\langle f \mid g\rangle=\sum_{z \in \mathcal{S}} \overline{f(z)} g(z)$. This space has vectors $v_{\alpha}$ as an orthonormal basis, where we define $v_{\alpha}\left(z_{\beta}\right)=\delta_{\alpha \beta}$. With respect to this basis, we may write functions in $\mathfrak{H}$ as $s$-dimensional complex vectors, so that $\langle f \mid g\rangle=f^{\dagger} g=\overline{f_{\alpha}} g_{\alpha}$, where we sum over the repeated index.

We will study model field theories derived from Lagrangians of form

$$
\mathcal{L}=\frac{i}{2}\left(\psi^{\dagger} \dot{\psi}-\dot{\psi}^{\dagger} \psi\right)-\psi^{\dagger} W \psi-\frac{\lambda}{2}\left(\psi^{\dagger} \psi\right)^{2}
$$

where $\psi(t) \in \mathfrak{H}, W$ is a self-adjoint positive operator on $\mathfrak{H}$ and $\lambda \in \mathbb{R}^{+}$. The corresponding field equation is

$$
\dot{\psi}=-i W \psi-i \lambda\left(\psi^{\dagger} \psi\right) \psi
$$

which conserves the quantity $\psi^{\dagger} \psi$, and therefore reduces to the linear equation

$$
\dot{\psi}=-i W \psi-i \lambda \psi^{\dagger}(0) \psi(0) \psi
$$

once the initial data $\psi(0)$ is specified. Thus the unique solution to Eq. (2.2) with this data is

$$
\psi(t)=e^{-i \lambda t \psi^{\dagger}(0) \psi(0)} e^{-i W t} \psi(0)
$$

The configuration space variables $\psi_{\alpha}$ have conjugate momenta $i \psi_{\alpha}^{\dagger}$, 2] and Eq. (2.2) may be written in the Hamiltonian form

$$
\dot{\psi}_{\alpha}=\frac{\partial h}{\partial\left(i \psi_{\alpha}^{\dagger}\right)},
$$

\footnotetext{
${ }^{1}$ Many of our results for free fields generalise easily to the case in which $\mathcal{S}$ is a manifold.

${ }^{2}$ Naïvely, one might expect the momenta to be $\frac{1}{2} i \psi_{\alpha}^{\dagger}$. However, the Lagrangian (2.1) is a second class constrained system and the correct momenta may be obtained using Dirac brackets [20].
} 
with Hamiltonian

$$
h\left(\psi, i \psi^{\dagger}\right)=\psi_{\alpha}^{\dagger} W_{\alpha \beta} \psi_{\beta}+\frac{\lambda}{2} \psi_{\alpha}^{\dagger} \psi_{\beta}^{\dagger} \psi_{\beta} \psi_{\alpha}
$$

To introduce CTC's we partition $\mathcal{S}$ into two subsets $\mathcal{S}_{1}$ and $\mathcal{S}_{2}$ containing $s_{1}$ and $s_{2}$ elements respectively, with $s_{1}+s_{2}=s$ and $s_{2} \leq s_{1}$, and make pointwise identifications of $\mathcal{S}_{2} \times\left\{T^{+}\right\}$with $\mathcal{S}_{2} \times\left\{0^{-}\right\}$and $\mathcal{S}_{2} \times\left\{T^{-}\right\}$with $\mathcal{S}_{2} \times\left\{0^{+}\right\}$for some $T>0$. This idealises wormholes linking the lower surface of $\mathcal{S}_{2}$ at $t=0$ with the upper surface of $\mathcal{S}_{2}$ at $t=T$, and the upper surface of $\mathcal{S}_{2}$ at $t=0$ with the lower surface of $\mathcal{S}_{2}$ at $t=T$. Note that $0^{-}$ and $0^{+}$(and correspondingly $T^{-}$and $T^{+}$) are regarded as distinct topological points for this purpose.

The partition of $\mathcal{S}$ induces a partition of the basis vectors $v_{\alpha}$ into the sets $e_{1}, \ldots, e_{s_{1}}$ and $f_{1}, \ldots, f_{s_{2}}$ whose respective spans are denoted $\mathfrak{H}_{1}$ and $\mathfrak{H}_{2}$. Clearly, we have $\operatorname{dim} \mathfrak{H}_{2} \leq \operatorname{dim} \mathfrak{H}_{1}$. We will also write the projection of $\psi \in \mathfrak{H}$ onto $\mathfrak{H}_{i}(i=1,2)$ as $\psi_{i}$. In the classical field theory, the identifications are implemented by the imposition of the boundary conditions

$$
\psi_{2}\left(T^{-}\right)=A \psi_{2}\left(0^{+}\right) \quad \text { and } \quad \psi_{2}\left(T^{+}\right)=B \psi_{2}\left(0^{-}\right)
$$

where $A, B$ are unitary maps of $\mathfrak{H}_{2}$ to itself, corresponding to the evolution through the wormholes (cf. Goldwirth et al. [6] $\left.\right|^{3}$ ). Politzer [1] takes $A=B=\mathbb{1}$. The rôle of these boundary conditions is simply to ensure that the evolution round the wormholes is consistent. We require $\psi_{1}(t)$ to be everywhere continuous, although $\dot{\psi}_{1}(t)$ may be discontinuous at $t=0, T$. Thus (2.2) is suspended at these points.

Except in the special cases in which $\mathcal{S}$ consists either of 2 points or 3 points arranged in a ring, the interaction term in (2.1) is not a nearest neighbour interaction and is therefore rather unsatisfactory as a model field theory. We will therefore restrict our discussion of specific interacting models to these cases. A more realistic field theory will be discussed elsewhere 21].

\section{THE CLASSICAL INITIAL VALUE PROBLEM}

In this section, we examine the behaviour of the classical field equation (2.2) subject to the CTC boundary conditions (2.7). For a generic class of $W$ and $T$, we show that the free field initial value problem is well posed for data specified before the dischronal region. We then examine the nonlinear theory and show that (generically) solutions exist for all initial data specified before the dischronal region; moreover, this solution is unique in the case of "weak coupling", but nonunique for "strong coupling".

To define the class of generic $W$ and $T$, we decompose the operator $e^{-i W T}$ (which implements the free evolution between $t=0^{+}$and $t=T^{-}$) in the block form

$$
e^{-i W T}=\left(\begin{array}{cc}
P & Q \\
R & S
\end{array}\right)
$$

\footnotetext{
${ }^{3}$ In the notation of [6], our $A$ is equal to $\left(W^{\downarrow}\right)^{-1}$, and $B$ is equal to $W^{\uparrow}$.
} 
with respect to the decomposition $\mathfrak{H}=\mathfrak{H}_{1} \oplus \mathfrak{H}_{2}$. The generic case is defined to be the case in which the norm $\|S\|$ of $S$ is strictly less than unity. (Note that $\|S\| \leq 1$ because $e^{-i W T}$ is unitary.) Equivalently, we require that $Q$ should be an injection from $\mathfrak{H}_{2}$ into $\mathfrak{H}_{1}$ so that $Q$ has a left inverse $K$, (i.e., such that $K Q=\left.\mathbb{1}\right|_{\mathfrak{H}_{2}}$ ) which is uniquely specified if we require it to annihilate the orthogonal complement of $\operatorname{Im} Q$. In this case we have $\|K\|=\|Q\|^{-1}$. This requirement is the reason for our restriction that $\operatorname{dim} \mathfrak{H}_{2} \leq \operatorname{dim} \mathfrak{H}_{1}$ : otherwise, $Q$ would necessarily have nontrivial kernel. The generic case corresponds to the situation expected in physically realistic field theories in which wavepackets spread out so that some proportion of any wave emerging from the wormhole at $t=0^{+}$manages to avoid reentering it at $t=T^{-}$.

\section{A. Free Case}

We now show that, in the generic case with $\lambda=0$, the equation of motion (2.2) with boundary conditions (2.7) constitutes a well posed initial value problem for arbitrary data $\psi \in \mathfrak{H}$ specified at $t=0^{-}$(and therefore for any $t<0$ ). In fact, we will only need the weaker condition that $A-S$ be invertible on $\mathfrak{H}_{2}$.

The evolution between $t=0^{+}$and $t=T^{-}$is given simply by the operator $e^{-i W T}$; accordingly, given data at $t=0^{-}$, the problem reduces to the study of the evolution between $t=0^{-}$and $t=0^{+}$. Because $\psi_{1}(t)$ is required to be continuous at $t=0$, it remains to determine $\psi_{2}\left(0^{+}\right)$in terms of $\psi\left(0^{-}\right)$. The only constraint on $\psi_{2}\left(0^{+}\right)$is that the CTC boundary conditions be satisfied, i.e., that $\psi_{2}\left(T^{-}\right)=A \psi_{2}\left(0^{+}\right)$. From Eq. (3.1) we have $\psi_{2}\left(T^{-}\right)=R \psi_{1}(0)+S \psi_{2}\left(0^{+}\right)$so, provided $A-S$ is invertible, $\psi_{2}\left(0^{+}\right)$is uniquely specified as

$$
\psi_{2}\left(0^{+}\right)=(A-S)^{-1} R \psi_{1}(0) .
$$

For $0<t<T$, the solution is thus

$$
\psi(t)=e^{-i W t}\left(\begin{array}{c}
\psi_{1}(0) \\
(A-S)^{-1} R \psi_{1}(0)
\end{array}\right),
$$

and in particular, we obtain

$$
\psi_{1}(T)=M \psi_{1}(0)
$$

where the matrix $M$ is

$$
M=P+Q(A-S)^{-1} R \text {. }
$$

The matrix $M$ is unitary, as we now show. Let $\psi \in \mathfrak{H}_{1}$ and use the unitarity of $e^{-i W T}$ to compute

\footnotetext{
${ }^{4}$ If $V_{1}$ and $V_{2}$ are complete normed linear spaces (Banach spaces) with norms $\|\cdot\|_{1}$ and $\|\cdot\|_{2}$ and $A$ : $V_{1} \rightarrow V_{2}$ is a linear mapping, then the norm $\|A\|$ of $A$ is defined by $\|A\|=\sup _{f \in V_{1} \backslash\{0\}}\|A f\|_{2} /\|f\|_{1}$.
} 


$$
\begin{aligned}
\|\psi\|^{2}+\left\|(A-S)^{-1} R \psi\right\|^{2} & =\left\|e^{-i W T}\left(\begin{array}{c}
\psi \\
(A-S)^{-1} R \psi
\end{array}\right)\right\|^{2} \\
& =\left\|\left(P+Q(A-S)^{-1} R\right) \psi\right\|^{2}+\left\|\left(\mathbb{1}+S(A-S)^{-1}\right) R \psi\right\|^{2} \\
& =\|M \psi\|^{2}+\left\|A(A-S)^{-1} R \psi\right\|^{2} .
\end{aligned}
$$

By unitarity of $A$, we now have $\|M \psi\|^{2}=\|\psi\|^{2}$ and conclude that $M$ is unitary.

Thus we have shown that there is a unique classical solution for each choice of initial data at $t=0^{-}$and that the full classical evolution from $t=0^{-}$to $t=T^{+}$is

$$
\psi\left(T^{+}\right)=\left(\begin{array}{cc}
M & 0 \\
0 & B
\end{array}\right) \psi\left(0^{-}\right) .
$$

Moreover, the solution is clearly continuous in the initial data, so we conclude that this initial value problem is well posed for data specified in the past of the CTC region on surfaces of constant $t$.

The situation is different for data specified between $t=0^{+}$and $t=T^{-}$. Here, the initial value problem is well posed only for a subclass of data satisfying certain consistency requirements. For example, data specified at $t=0^{+}$must obey Eq. (3.2). This phenomenon has been noted before in various situations [4.6,22]; it arises because the CTC's introduce constraints on the dynamics and has important implications for the quantum theory. Note that one may nonetheless specify the data at any given point freely: it is always possible to choose the remaining initial data so as to satisfy the consistency requirements. Thus our system has a "benignity" property analogous to those discussed in [4,22]. Related to this phenomenon is the fact that the classical evolution is nonunitary between $t=0^{-}$and $0^{+}$ and between $t=T^{-}$and $T^{+}$. To see this, take any initial data with $\psi_{1}(0)=0$ : at $t=0^{-}$, the initial data has norm $\left\|\psi_{2}\left(0^{-}\right)\right\|$; for $0<t<T$ the solution vanishes identically; and finally, at $t=T^{+}$, the solution again has norm $\left\|\psi_{2}\left(0^{-}\right)\right\|$.

Finally, it is instructive to see how this classical evolution may be derived using the path integral methods of Goldwirth et al. [6]. This is described in Appendix A.

\section{B. Interacting Case}

We now consider the full interacting classical field theory in the generic case. We will show: (i) there exists at least one solution for arbitrary initial data; (ii) there is a weak coupling regime in which there is a unique solution; and (iii) there is a strong coupling regime in which there exist many distinct solutions for each choice of initial data.

In the absence of CTC boundary conditions, the solution is given by (2.4). We write $a=\psi_{1}(0)$ and $b=\psi_{2}\left(0^{+}\right)$and implement the CTC boundary conditions by requiring $b$ to satisfy

$$
A b=e^{-i \lambda T\left(a^{\dagger} a+b^{\dagger} b\right)}(R a+S b)
$$

for given $a$.

To study the solutions to this equation, we first note that it implies $\|b\|=\|R a+S b\|$

and hence, by the unitarity of $e^{-i W T}$, that $\|a\|=\|P a+Q b\|$. In the generic case (in which $Q$ has left inverse $K$ ) any solution $b$ must therefore take the form 


$$
b=K(U-P) a,
$$

for some unitary $U$ on $\mathfrak{H}_{1}$. Substituting back into Eq. (3.8), and rearranging, we find that $b$ solves (3.8) if and only if

$$
K U a=K f(U) a
$$

where $f(U)=P+Q\left(A e^{i \eta(U)}-S\right)^{-1} R$ and

$$
\eta(U)=\lambda T a^{\dagger}\left\{\mathbb{1}+(U-P)^{\dagger} K^{\dagger} K(U-P)\right\} a .
$$

Because $\eta(U)$ is real-valued, $f(U)$ is a unitary operator on $\mathfrak{H}_{2}$.

Clearly, any solution to the fixed point equation $U=f(U)$ necessarily yields a solution to Eq. (3.8); moreover, any such $U$ must take the form $U(z)=P+Q(z A-S)^{-1} R$ for some $z$ on the unit circle. (Note that this expression is always well-defined because $\|S\|<1$.) Thus the problem of existence reduces to finding fixed points of the equation $z=e^{i \eta(U(z))}$ on the unit circle. Now

$$
\eta(U(z))=\lambda T\left(\|a\|^{2}+\left\|(z A-S)^{-1} R a\right\|^{2}\right),
$$

which is a continuous single-valued function from the unit circle to the real line; thus $e^{i \eta(U(z))}$ is a mapping of the unit circle to itself with vanishing Brouwer degree (see, e.g., Ch. 1 of [23]). Accordingly, for each choice of initial data $a \in \mathfrak{H}_{1}$ there exists at least one fixed point of $g$ and thus at least one solution to Eq. (3.8), so we have proved the claim (i) above.

To establish claim (ii), we write the right hand side of Eq. (3.8) as $A g(b)$ where $g: \mathfrak{H}_{2} \rightarrow$ $\mathfrak{H}_{2}$ and consider the fixed point problem $b=g(b)$ on the ball $\mathcal{B}=\left\{b \in \mathfrak{H}_{2} \mid\|b\| \leq r_{0}\|a\|\right\}$, where $r_{0}=\|Q\|^{-1}(1+\|P\|)$. This ball contains all solutions to Eq. (3.8) as a consequence of Eq. (3.9). For any $b_{1}, b_{2}$ in $\mathcal{B}$, we have

$$
\begin{aligned}
\left\|g\left(b_{1}\right)-g\left(b_{2}\right)\right\|= & \|\left(1-e^{i \lambda T\left(\left\|b_{1}\right\|^{2}-\left\|b_{2}\right\|^{2}\right)}\right)\left(R a+S b_{1}\right) \\
& +e^{i \lambda T\left(\left\|b_{1}\right\|^{2}-\left\|b_{2}\right\|^{2}\right)} S\left(b_{1}-b_{2}\right) \| \\
\leq & \left(c_{0} \lambda T\|a\|^{2}+\|S\|\right)\left\|b_{1}-b_{2}\right\|
\end{aligned}
$$

in which we have used the elementary estimates $\left|1-e^{i \alpha}\right| \leq|\alpha|$ and $\left|\left\|b_{1}\right\|-\left\|b_{2}\right\|\right| \leq\left\|b_{1}-b_{2}\right\|$, and $c_{0}=2 r_{0}\left(\|R\|+\|S\| r_{0}\right)$ is a positive real constant depending only on $P, Q, R$ and $S$. In the generic case, for $\lambda T\|a\|^{2}<c_{0}^{-1}(1-\|S\|)$ (i.e., weak coupling), $\left.g\right|_{\mathcal{B}}$ is a strict contraction (which need not map $\mathcal{B}$ to itself) and standard contraction mapping arguments now imply that there can be at most one fixed point in $\mathcal{B}$. Putting this together with (i) and using the fact that all fixed points of $g$ must lie in $\mathcal{B}$, we have proved (ii).

Finally, to prove (iii) we note that if $\lambda T\|R a\|^{2} \gg 1$ (i.e., strong coupling) then the fixed point problem $z=e^{i \eta(U(z))}$ described above has many solutions on the unit circle; moreover, because $R a \neq 0$, these solutions must correspond to distinct values of $b$ and hence of $\psi_{1}(T)$.

In Figs. 1 and 2, we explicitly show how nonuniqueness arises in a model with two spatial points and $P=-Q=R=S=1 / \sqrt{2}$ with $A=\mathbb{1}$. For this model the classical solutions are in one-to-one correspondence with the solutions of the fixed point equation $z=e^{i \eta(U(z))}$ on the unit circle, because $K$ and $U(z)$ are scalars. Writing $z=e^{i \theta}$, this becomes 


$$
\zeta(\theta)=\theta+2 k \pi,
$$

for $k \in \mathbb{Z}$ where $\zeta(\theta)=\mu\left(1+\left|\sqrt{2} e^{i \theta}-1\right|^{-2}\right)$ and we have written $\mu=\lambda T|a|^{2}$ for the "coupling strength". This equation may be solved graphically by plotting each side separately for $-\pi<\theta \leq \pi$ looking for intersections. Fig. 1 shows the appropriate plots for $\mu=0.5$, from which it is clear that there is a unique solution, whilst Fig. 2 corresponds to the case $\mu=3.0$ where there are 7 solutions.

\section{Preservation of the Symplectic Structure}

Except at $t=0, T$, the classical dynamics is generated by the Hamiltonian $h$, and therefore preserves the symplectic structure on phase space in the initial and final chronal regions. Owing to the CTC boundary conditions, it is not clear that the evolution from initial to final chronal regions also preserves the symplectic structure. Here, we express the classical evolution in phase space language and prove that the evolution from initial to final chronal regions is implemented by a symplectic transformation.

The classical phase space is $\Gamma=\mathbb{C}^{s}$ with symplectic structure given by the 2 -form $\Omega=-i d \psi^{\dagger} \wedge d \psi=-i d \bar{\psi}_{\alpha} \wedge d \psi_{\alpha}$. In the usual way, functions on $\Gamma$ are regarded as functions of independent variables $\psi$ and $\psi^{\dagger}$. A symplectic transformation $\xi$ is a diffeomorphism of $\Gamma$ which preserves $\Omega$, i.e., $\xi^{*} \Omega=\Omega$, where $\xi^{*} \Omega$ is the pull-back of $\Omega$ by $\xi$. This is equivalent (see e.g., $§ 40$ in [24]) to the Dirac bracket relation

$$
\{f \circ \xi, g \circ \xi\}_{D, x}=\{f, g\}_{D, \xi(x)},
$$

where the Dirac bracket of two functions on $\Gamma$ is defined by

$$
\{f, g\}_{D, x}=\left.\left(\frac{\partial f}{\partial \psi_{\gamma}} \frac{\partial g}{\partial i \bar{\psi}_{\gamma}}-\frac{\partial g}{\partial \psi_{\gamma}} \frac{\partial f}{\partial i \bar{\psi}_{\gamma}}\right)\right|_{x} .
$$

Corresponding to the decomposition $\psi=\left(\psi_{1}, \psi_{2}\right)$, we have $\Gamma=\Gamma_{1} \times \Gamma_{2}$, and associated natural projections $\pi_{k}: \Gamma \rightarrow \Gamma_{k}$. Then $\Omega$ can be expressed as

$$
\Omega=\pi_{1}^{*} \Omega_{1}+\pi_{2}^{*} \Omega_{2},
$$

where, for $k=1,2, \Omega_{k}=-i d \psi_{k}^{\dagger} \wedge d \psi_{k}$ is the symplectic form on $\Gamma_{k}$. Any unitary matrix $U$ on $\mathfrak{H}_{2}$ defines a corresponding natural symplectic transformation of $\Gamma_{2}$, which we denote $\chi_{U}$. In addition, for $t \in \mathbb{R}, \tau_{t}=\exp t I d h$ is the evolution generated by the Hamiltonian $h$, where $I$ is the canonical isomorphism between 1 -forms and vector fields on $\Gamma$ specified by $\Omega(I \omega, \cdot)=\omega(\cdot)$. We have $\tau_{t}^{*} \Omega=\Omega$ for all $t$.

With these definitions, the diffeomorphism $\eta$ implementing evolution from $t=0^{-}$to $t=T^{+}$is $\eta=\left(\kappa, \chi_{B}\right)$, where $B$ is the unitary matrix appearing in the CTC boundary conditions and $\kappa$ is a mapping from an open set $U \subset \Gamma_{1}$ into $\Gamma_{1}$ defined as follows. First, we define a differentiable map $\sigma: U \rightarrow \Gamma$ as a solution to the equations

$$
\pi_{1} \circ \sigma=\mathrm{id}_{1}, \quad \pi_{2} \circ \tau_{T} \circ \sigma=\chi_{A} \circ \pi_{2} \circ \sigma
$$

which express the classical consistency requirement Eq. (3.8). Then $\kappa$ is defined by 


$$
\kappa=\pi_{1} \circ \tau_{T} \circ \sigma
$$

In general, there will be many possible choices for $\kappa$ reflecting the nonuniqueness of the classical evolution. For any such choice, the relation $\kappa^{*} \Omega_{1}=\Omega_{1}$ can be proved using the composition rule $(f \circ g)^{*}=g^{*} f^{*}$ as

$$
\begin{aligned}
\kappa^{*} \Omega_{1} & =\sigma^{*} \tau_{T}^{*} \pi_{1}^{*} \Omega_{1} \\
& =\sigma^{*} \tau_{T}^{*}\left(\Omega-\pi_{2}^{*} \Omega_{2}\right) \\
& =\sigma^{*} \Omega-\sigma^{*} \tau_{T}^{*} \pi_{2}^{*} \Omega_{2} \\
& =\sigma^{*}\left(\pi_{1}^{*} \Omega_{1}+\pi_{2}^{*} \Omega_{2}\right)-\sigma^{*} \pi_{2}^{*} \chi_{B}^{*} \Omega_{2} \\
& =\Omega_{1} .
\end{aligned}
$$

Thus, because $\chi_{B}^{*} \Omega_{2}=\Omega_{2}$, we conclude that $\eta=\left(\kappa, \chi_{B}\right)$ preserves $\Omega$.

In terms of Dirac brackets, writing $\psi\left(T^{+}\right)=\eta\left(\psi, i \psi^{\dagger}\right)$, we have proved

$$
\left\{\psi_{\alpha}\left(T^{+}\right), \psi_{\beta}\left(T^{+}\right)\right\}_{D}=0
$$

and

$$
\left\{\psi_{\alpha}\left(T^{+}\right), \bar{\psi}_{\beta}\left(T^{+}\right)\right\}_{D}=-i \delta_{\alpha \beta}
$$

Note that the evolution between $t=0^{-}$and $t=0^{+}$(and similarly between $t=T^{-}$and $\left.t=T^{+}\right)$is not symplectic in general.

\section{THE QUANTUM INITIAL VALUE PROBLEM IN THE ABSENCE OF CTC'S}

In order to prepare for our discussion of chronology violating models, it is useful to show how a study of the QIVP reproduces the results of canonical quantisation for Eq. (2.2) in the absence of CTC's. We first discuss the case of Fermi statistics to avoid the operator domain technicalities of the bosonic case.

The canonical approach starts by identifying the classical canonical coordinates $\psi_{\alpha}$ and $i \psi_{\alpha}^{\dagger}$ and the classical Hamiltonian $h\left(\psi_{\alpha}, i \psi_{\alpha}^{\dagger}\right)$ defined in Eq. (2.6). A Hilbert space $\mathfrak{F}$ is then constructed on which bounded operators $\Psi_{1}, \ldots, \Psi_{s}$ represent the CAR's for $s$ degrees of freedom - that is, $\left\{\Psi_{\alpha}, \Psi_{\beta}\right\}=0$ and $\left\{\Psi_{\alpha}, \Psi_{\beta}^{\dagger}\right\}=\delta_{\alpha \beta}$ for all $\alpha, \beta$. The quantised (normal ordered) Hamiltonian $H$ is defined as a (bounded) self-adjoint operator on $\mathfrak{F}$ by substituting $\Psi_{\alpha}$ for $\psi_{\alpha}$ in the RHS of Eq. (2.6) using its literal ordering. The quantum evolution generated by $H$ evolves a general operator $A$ from time 0 to $t$ by

$$
A(t)=e^{i H t} A e^{-i H t},
$$

and the evolved operator therefore satisfies the Heisenberg equation of motion

$$
\dot{A}(t)=i[H, A(t)] .
$$

Thus, by virtue of the CAR's, $\Psi_{\alpha}(t)=e^{i H t} \Psi_{\alpha} e^{-i H t}$ solves the original equation of motion (2.2) as an operator differential equation with initial data $\Psi_{\alpha}(0)=\Psi_{\alpha}$. Moreover, the CAR's are necessarily preserved by this evolution. 
It is possible to reproduce these results from a slightly different angle, namely by treating Eq. (2.2) as an operator differential equation and considering the quantum initial value problem (QIVP). Given initial data $\Psi_{\alpha}$ representing the CAR's, we say that the QIVP is well posed if there exists a unique operator-valued solution $\Psi_{\alpha}(t)$ to Eq. (2.2) with $\Psi_{\alpha}(0)=\Psi_{\alpha}$ and the evolution preserves the CAR's. To show that this is indeed the case, we note that for arbitrary initial data given as bounded operators on $\mathfrak{F}$, Eq. (2.2) has the unique solution

$$
\Psi_{\alpha}(t)=e^{-i \lambda t \Psi_{\gamma}^{\dagger} \Psi_{\gamma}}\left(e^{-i W t}\right)_{\alpha \beta} \Psi_{\beta}
$$

The proof of uniqueness closely parallels the analogous argument for the classical differential equation. One may check that this evolution preserves the CAR's either by explicit computation or by noting that the above solution must agree (by uniqueness) with that obtained from the canonical approach. Thus the QIVP for Eq. (2.2) is well posed in the fermionic case.

Of course, it is not usually advantageous to consider the QIVP directly because it is rare that the equation of motion may be solved in closed form for general operator-valued initial data. However, for the chronology violating models considered in this paper, it will not always be possible to assume that the initial data is a representation of the canonical (anti)commutation relations and therefore the canonical method is no longer guaranteed to yield solutions to the equation of motion Eq. (2.2). In these situations, we must therefore employ the more general setting of the QIVP.

In the bosonic case, we encounter unbounded operators and therefore must proceed more carefully. We now describe the technicalities required in order to generalise the foregoing to this case.p

Definition Let $\mathcal{D}$ be dense in Hilbert space $\mathfrak{F}$, and let $\Psi_{1}(t), \ldots, \Psi_{s}(t)$ be closed operatorvalued functions on $\mathbb{R}$ such that $\mathcal{D}$ is a core for each $\Psi_{\alpha}(t)$ and is invariant under the $\Psi_{\alpha}(t)$ and $\Psi_{\alpha}^{\dagger}(t)$. Then the $\Psi_{\alpha}(t)$ are said to be a solution to $E q$. (2.2) on $\mathcal{D}$ if each $\Psi_{\alpha}(t)$ is strongly differentiable with respect to $t$ on $\mathcal{D}$ with derivative $-i W_{\alpha \beta} \Psi_{\beta}(t)-i \lambda \Psi_{\gamma}(t)^{\dagger} \Psi_{\gamma}(t) \Psi_{\alpha}(t)$.

Note that this definition extends that used above for the bounded case.

Definition The closed operators $\Psi_{1}, \ldots, \Psi_{s}$ are said to represent the CCR's on $\mathfrak{F}$ if they have a common dense domain $\mathcal{X}$ invariant under both the $\Psi_{\alpha}$ and the $\Psi_{\alpha}^{\dagger}$ with

$$
\left[\Psi_{\alpha}, \Psi_{\beta}\right] f=\left[\Psi_{\alpha}^{\dagger}, \Psi_{\beta}^{\dagger}\right] f=0
$$

and

\footnotetext{
${ }^{5}$ For completeness, we give the following definitions (see [25]). An operator $A$ with dense domain $D(A)$ in Hilbert space $\mathfrak{F}$ is closed if its graph (i.e., the set of pairs $\langle f, A f\rangle$ as $f$ runs through $D(A)$ ) is a closed subspace of $\mathfrak{F} \times \mathfrak{F}$ in the product topology; $A$ is closable if the closure of its graph is itself the graph of an operator, called the closure of $A$. An algebraic subspace $\mathcal{D}$ of $\mathfrak{F}$ contained in $D(A)$ is a core for a closed operator $A$ if $A$ is the closure of its restriction to $\mathcal{D}$. A densely defined operator $A$ is essentially self-adjoint if its closure is self-adjoint. $\mathcal{D} \subset D(A)$ is invariant under $A$ if $A f \in \mathcal{D}$ for all $f \in \mathcal{D}$. Finally, an operator-valued function $A(t)$ is strongly differentiable with respect to $t$ on $\mathcal{D}$ with derivative $B(t)$ if $\mathcal{D}$ is contained in $D(B(t))$ and $D(A(\tau))$ for all $\tau$ in some neighbourhood of $t$ and $\left\|\left(\epsilon^{-1}(A(t+\epsilon)-A(t))-B(t)\right) f\right\| \rightarrow 0$ as $\epsilon \rightarrow 0$ for all $f \in \mathcal{D}$.
} 


$$
\left[\Psi_{\alpha}, \Psi_{\beta}^{\dagger}\right] f=\delta_{\alpha \beta} f
$$

for all $f \in \mathcal{X}$ and such that $\Psi_{\alpha}^{\dagger} \Psi_{\alpha}$ (summing over the repeated index) is decomposable on $\mathcal{X}$. That is, there exists a projection-valued measure $P_{\Omega}$ on $\mathbb{R}$ such that $\mathcal{X}$ contains $\mathcal{D}_{0}=\bigcup_{\mu \geq 0} P_{[-\mu, \mu]} \mathfrak{F}$ and $\Psi_{\alpha}^{\dagger} \Psi_{\alpha} f=\int_{\mathbb{R}} \mu d P_{\mu} f$ for all $f \in \mathcal{X}$.

The reason for the technical requirement of decomposability is that it guarantees [26] that all such representations of the CCR's are equivalent up to unitary equivalence and multiplicity (i.e., the conclusion of von Neumann's theorem holds).

The canonical quantisation of Eq. (2.2) proceeds as follows. Suppose that operators $\Psi_{\alpha}$ represent the CCR's on Hilbert space $\mathfrak{F}$ with dense invariant domain $\mathcal{X}$, and let $\mathcal{D}_{0} \subset \mathcal{X}$ be defined as above. The quantum Hamiltonian may be defined on $\mathcal{D}_{0}$ by substituting the operators $\Psi_{\alpha}$ into the RHS of Eq. (2.6) to yield an essentially self-adjoint operator whose closure is denoted by $H$. Moreover, $\mathcal{D}_{0}$ is easily seen to be invariant under $e^{i H t}$ for $t \in \mathbb{R}$. Thus, the evolved operators $\Psi_{\alpha}(t)$ defined by

$$
\Psi_{\alpha}(t)=e^{i H t} \Psi_{\alpha} e^{-i H t}
$$

are strongly differentiable with respect to $t$ on $\mathcal{D}_{0}$ with derivative $i e^{i H t}\left[H, \Psi_{\alpha}\right] e^{-i H t}$ and the CCR's may then be used (on $\mathcal{D}_{0}$ ) to conclude that the $\Psi_{\alpha}(t)$ solve Eq. (2.2) on $\mathcal{D}_{0}$ in the sense defined above.

As in the fermionic case, we may reproduce these results by studying the QIVP. The situation for general initial data is summarised by the following:

Proposition Let $\mathfrak{F}$ be a Hilbert space and $\mathcal{D} \subset \mathfrak{F}$ be dense. Suppose that $\Psi_{\alpha},(\alpha=1, \ldots, s)$ are closed (possibly unbounded) operators on $\mathfrak{F}$ such that

(i) $\mathcal{D}$ is a core for each $\Psi_{\alpha}$ and a domain of essential self-adjointness for $\Psi_{\gamma}^{\dagger} \Psi_{\gamma}$

(ii) $\mathcal{D}$ is invariant under $\Psi_{\alpha}, \Psi_{\alpha}^{\dagger}$ and $e^{-i \lambda t \Psi_{\gamma}^{\dagger} \Psi_{\gamma}}$ for all $t \in \mathbb{R}$.

Then the operators $\Psi_{\alpha}(t)$ defined as the closure of $e^{-i \lambda t \Psi_{\gamma}^{\dagger} \Psi_{\gamma}}\left(e^{-i W t}\right)_{\alpha \beta} \Psi_{\beta}$ on $\mathcal{D}$ constitute the unique solution to Eq. (2.9) on $\mathcal{D}$ with initial data $\Psi_{\alpha}$.

An immediate corollary of this is that if the $\Psi_{\alpha}$ represent the CCR's on $\mathfrak{F}$ and the domain $\mathcal{D}_{0}$ is defined as above, then the QIVP for Eq. (2.2) is well posed on $\mathcal{D}_{0}$.

\section{THE QUANTUM INITIAL VALUE PROBLEM FOR CHRONOLOGY VIOLATING MODELS}

\section{A. General Formalism}

We now analyse the quantum initial value problem for Eq. (2.2) in the presence of CTC's, beginning with the case of the CAR's. Suppose that the operators $\Psi_{\alpha}(\alpha=1, \ldots, s)$ provide a representation of the CAR's on Hilbert space $\mathfrak{F}$. We specify these operators as the initial data for the QIVP at time $t=0^{-}$. Writing $\Psi_{1, i}$ and $\Psi_{2, j}$ to denote those operators associated with $\mathcal{S}_{1}$ and $\mathcal{S}_{2}$ respectively, we therefore seek operators $\Psi_{2, j}\left(0^{+}\right)$such that the evolution between $t=0^{+}$and $T^{-}$obeys the consistency requirement $\Psi_{2, i}\left(T^{-}\right)=A_{i j} \Psi_{2, j}\left(0^{+}\right)$. Denoting $\Psi_{1, i}=a_{i}, \Psi_{2, j}\left(0^{+}\right)=b_{j}$ we therefore require the $b_{i}$ to satisfy 


$$
A_{i j} b_{j}=e^{-i \lambda T\left(a_{k}^{\dagger} a_{k}+b_{k}^{\dagger} b_{k}\right)}\left(R_{i j} a_{j}+S_{i j} b_{j}\right) .
$$

Remarkably, and in contrast to the situation for the classical theory, it turns out that this specifies the $b_{i}$ uniquely in the generic case as we now show.

We first construct a solution to Eq. (5.1) and then prove its uniqueness. For $z \in \mathbb{C}$, let $N(z)_{i j}$ be the matrix-valued function of $z$ defined by $N(z)=(z A-S)^{-1} R$, which is analytic in an open neighbourhood of the unit circle in the generic case. Then for any unitary operator $V$ on Hilbert space $\mathfrak{K}$, we may use the (Dunford) functional calculus (see e.g., pp. 556-577 of [27]) to define $N(V)_{i j}$ as a matrix of bounded operators on $\mathfrak{K}$. Using this notation, Eq. (5.1) may be rewritten in the form

$$
b_{i}=N\left(e^{i \lambda T\left(a_{k}^{\dagger} a_{k}+b_{k}^{\dagger} b_{k}\right)}\right)_{i j} a_{j} .
$$

Next, let $\mathfrak{F}_{r}$ be the eigenspace of $a_{i}^{\dagger} a_{i}$ with eigenvalue $r$ and decompose $\mathfrak{F}=\bigoplus_{r} \mathfrak{F}_{r}$. We emphasise that $a_{i}^{\dagger} a_{i}$ is not the total particle number on $\mathcal{S}$ at $t=0^{-}$, but rather the particle number on $\mathcal{S}_{1}$. Thus, for example, $\mathfrak{F}_{0}$ is not 1-dimensional, but consists of all states at $t=0^{-}$ with no $\mathcal{S}_{1}$-particles. We now define unitary operators $U_{r}$ on the $\mathfrak{F}_{r}$ by the recurrence relation

$$
U_{r+1}=\exp i \lambda T a_{i}^{\dagger}\left(\delta_{i k}+N\left(U_{r}\right)_{i j}^{\dagger} N\left(U_{r}\right)_{j k}\right) a_{k},
$$

with $U_{0}=\mathbb{1}$. Denoting $U=\bigoplus_{r} U_{r}$, it is easy to see that Eq. (5.1) is solved by

$$
b_{i}=N(U)_{i j} a_{j}
$$

by comparing with Eq. (5.2) and using the fact that each $a_{i}$ maps $\mathfrak{F}_{r+1}$ to $\mathfrak{F}_{r}$ and annihilates $\mathfrak{F}_{0}$.

We now prove that (5.4) is the unique solution to Eq. (5.1). Suppose that $b_{1}, \ldots, b_{s_{2}}$ solve Eq. (5.1), and write $U=e^{i \lambda T\left(a_{k}^{\dagger} a_{k}+b_{k}^{\dagger} b_{k}\right)}$. Because $N(U)_{i j}$ is a matrix of bounded operators, Eq. (5.2) implies that the $b_{i}$ annihilate $\mathfrak{F}_{0}$. Accordingly, $U$ leaves $\mathfrak{F}_{0}$ invariant and $\left.U\right|_{\mathfrak{F}_{0}}=\mathbb{1}$. Now suppose inductively that $U$ leaves $\mathfrak{F}_{r}$ invariant for some $r \geq 0$. Provided that $r$ is not the largest eigenvalue of $a_{k}^{\dagger} a_{k}$, Eq. (5.2) and its adjoint imply that each $b_{i}$ maps $\mathfrak{F}_{r+1}$ to $\mathfrak{F}_{r}$ and each $b_{i}^{\dagger}$ maps $\mathfrak{F}_{r}$ to $\mathfrak{F}_{r+1}$. Accordingly $a_{k}^{\dagger} a_{k}+b_{k}^{\dagger} b_{k}$ and thus $U$ leave $\mathfrak{F}_{r+1}$ invariant. Hence by induction, we find that each $\mathfrak{F}_{r}$ is an invariant subspace for $U$, so we may write $U=\bigoplus_{r} U_{r}$ with each $U_{r}$ unitary on $\mathfrak{F}_{r}$. It is then easy to see that the $U_{r}$ must satisfy the recurrence relation (5.3) with $U_{0}=\left.U\right|_{\mathfrak{F}_{0}}=\mathbb{1}$. We have therefore completed the proof of uniqueness.

Finally, we note that this solution is representation independent in the following sense. Suppose that $\Psi_{\alpha}$ form a Fock representation of the CAR's, and let $b_{i}$ be the unique solution to Eq. (5.1) on $\mathfrak{F}$. By the Jordan-Wigner theorem (see e.g., [28]), an arbitrary representation $\Psi_{\alpha}^{\prime}$ on $\mathfrak{F}^{\prime}$ takes the form $\Psi_{\alpha}^{\prime}=U^{-1}\left(\Psi_{\alpha} \otimes \mathbb{1}\right) U$, where $U: \mathfrak{F}^{\prime} \rightarrow \mathfrak{F} \otimes \mathfrak{N}$ is unitary and $\mathfrak{N}$ is an auxiliary Hilbert space. Then the unique solution to the analogue of Eq. (5.1) on $\mathfrak{F}^{\prime}$ is $b_{i}^{\prime}=U^{-1}\left(b_{i} \otimes \mathbb{1}\right) U$.

In the CCR case, certain domain questions must be addressed. We suppose that the $\Psi_{\alpha}$ are a representation of the CCR's on $\mathfrak{F}$ with common invariant domain $\mathcal{X}$ and define $\mathcal{D}_{0} \subset \mathcal{X}$ as in Section $\mathbb{V}$. An important property of this domain is that $\mathfrak{F}_{r} \subset \mathcal{D}_{0}$ for all $r$, where $\mathfrak{F}_{r}$ is again defined as the eigenspace of $a_{i}^{\dagger} a_{i}$ with eigenvalue $r$. Then it is easy to see 
that the same construction as used in the CAR case yields a solution to Eq. (5.1) on $\mathcal{D}_{0}$; moreover, one may show that it is the unique solution such that $\mathcal{D}_{0}$ is a core for each $b_{i}$, and is independent of representation in the same sense as in the CAR case.

Once the unique solution to Eq. (5.1) has been obtained (for either CAR's or CCR's) we may substitute back to find

$$
a_{i}(T)=e^{-i \lambda T\left(a_{k}^{\dagger} a_{k}+b_{k}^{\dagger} b_{k}\right)}\left(P_{i j} a_{j}+Q_{i j} b_{j}\right),
$$

and check to see whether or not this evolution preserves the CCR/CAR's and is therefore unitary. We will analyse various cases of this problem in the following subsections.

\section{B. Free Fields}

Here $\lambda=0$ and Eq. (5.2) immediately yields the unique solution

$$
b_{i}=(A-S)_{i j}^{-1} R_{j k} a_{k} .
$$

Substituting, we find that the evolution is given by

$$
\begin{aligned}
\Psi_{1, i}(T) & =M_{i j} \Psi_{1, j}(0) \\
\Psi_{2, i}\left(T^{+}\right) & =B_{i j} \Psi_{2, j}\left(0^{-}\right),
\end{aligned}
$$

where $M=P+Q(A-S)^{-1} R$ is unitary. Note that one obtains the same result for both Bose and Fermi statistics. This evolution is easily seen to preserve the CCR/CAR's; there is therefore a unitary $X$ on $\mathfrak{F}$ such that

$$
\Psi_{\alpha}\left(T^{+}\right)=X^{\dagger} \Psi_{\alpha}\left(0^{-}\right) X .
$$

An interesting feature of the above is that the operators $b_{i}$ are linearly dependent on the $a_{i}$. Thus the components of $\Psi\left(0^{+}\right)$do not form a representation of the CCR/CAR's for $M+N$ degrees of freedom. In effect the system is reduced to only $M$ degrees of freedom, reflecting the fact that the CTC's place $N$ constraints on the system. Accordingly, the evolution between $t=0^{-}$and $t=0^{+}$is nonunitary, although unitarity is restored at $t=T^{+}$. In addition, we see that it is not legitimate to employ canonical methods to evolve the quantum field in the dischronal region (if one intends to solve the equation of motion Eq. (2.2)) because the data at $t=0^{+}$does not obey the CCR/CAR's.

As a final check on our result in this case, and on the loss of degrees of freedom, let us quantise by the familiar method of obtaining classical mode solutions. Let $e_{i}(t)$ (respectively, $f_{j}(t)$ ) be the classical solution to the free equation of motion with initial data $e_{i}\left(0^{-}\right)=e_{i}$ $\left(f_{j}\left(0^{-}\right)=f_{j}\right)$ where the basis vectors $e_{i}$ and $f_{j}$ were defined in Sect. [1]. We write the quantum field $\Psi(t)$ as

$$
\Psi(t)=a_{i} e_{i}(t)+b_{j} f_{j}(t),
$$

where the $a_{i}$ and $b_{j}$ form a representation of the CCR/CAR's on Hilbert space $\mathfrak{F}$. The components $\Psi_{\alpha}$ of the field are obtained by taking the inner product with $v_{\alpha}$. The time evolution of the $a_{i}$ and $b_{i}$ is defined by re-expressing the field as 


$$
\Psi(t)=a_{i}(t) e_{i}+b_{j}(t) f_{j}
$$

which leads quickly to the above unitary evolution from $0^{-}$to $T^{+}$using the results of Section III. In the dischronal region, however, $f_{j}(t)$ vanishes and so $\Psi(t)=a_{i} e_{i}(t)$ and the reduction to $M$ degrees of freedom is explicit.

\section{Interacting Fields}

Here, we consider three simple examples. Model 1 is a system with two spatial points and yields a unitary theory for both Fermi and Bose statistics. Model 2 is a system with three spatial points. We study this theory for Fermi statistics and show that the resulting theory is nonunitary. For simplicity we work in the appropriate Fock representations and take $A$ and $B$ to be the identity.

Model 1 Our set of spatial points is $\mathcal{S}=\left\{z_{1}, z_{2}\right\}$, and $\mathcal{S}_{i}=\left\{z_{i}\right\}$ for $i=1,2$. Thus $W$ is a $2 \times 2$ matrix and $P, Q, R, S$ are scalars.

Fermi statistics The Hilbert space $\mathfrak{F}$ for two fermionic degrees of freedom is isomorphic to $\mathbb{C}^{2} \otimes \mathbb{C}^{2}$. The unique solution to Eq. (5.1) is

$$
b=(1-S)^{-1} R a,
$$

as is easily verified using the fact that $e^{i \kappa a^{\dagger} a} a=a$. Substituting back, we obtain

$$
a(T)=\left(P+Q(1-S)^{-1} R\right) a,
$$

which is identical to the unitary free evolution obtained in the previous subsection. This contrasts with the generically nonunitary evolution obtained by Politzer [1] for this model using the self-consistent path integral - see Section IX].

Bose Statistics Here, $\mathfrak{F}=\ell^{2} \otimes \ell^{2}$ (where $\ell^{2}$ is the Hilbert space of square summable sequences) and the unique solution to Eq. (5.1) takes the form

$$
b=f\left(a^{\dagger} a\right) a
$$

where $f: \mathbb{N} \rightarrow \mathbb{C}$ is defined recursively by

$$
f(n+1)=\left(e^{i \lambda T(n+1)\left(1+|f(n)|^{2}\right)}-S\right)^{-1} R,
$$

with $f(0)=(1-S)^{-1} R$.

Thus the evolution of $a$ is given by

$$
\begin{aligned}
a(T) & =e^{-i \lambda T a^{\dagger}\left(1+\left|f\left(a^{\dagger} a\right)\right|^{2}\right) a}\left(P+Q f\left(a^{\dagger} a\right)\right) a \\
& =e^{-i g\left(a^{\dagger} a\right)}\left(P+Q\left(e^{i g\left(a^{\dagger} a\right)}-S\right)^{-1} R\right) a,
\end{aligned}
$$

where $g$ is a real-valued function on $\mathbb{N}$ defined by $g(0)=0$ and $g(n)=\lambda \operatorname{Tn}\left(1+|f(n-1)|^{2}\right)$ for $n \geq 1$. This may be rewritten as

$$
a(T)=X^{\dagger} a X
$$


with $X=e^{-i h\left(a^{\dagger} a\right)}$ and $h(n)$ defined by $h(0)=0$ and

$$
\begin{aligned}
e^{-i h(n+1)}= & e^{-i h(n)} e^{-i g(n)} \\
& \times\left[P+Q\left(e^{i g(n)}-S\right)^{-1} R\right] .
\end{aligned}
$$

The left hand side is always of unit modulus, so $h(n)$ is real-valued and the operator $X$ is unitary. Thus the evolution from $t=0^{-}$to $t=T^{+}$is again unitary. We note that this theory agrees with the corresponding free theory on $\mathfrak{F}_{1}$ (though the theories differ on $\mathfrak{F}_{r}$ for $r \geq 2$ ).

Model 2 In this example, our set of spatial points $\mathcal{S}=\left\{z_{1}, z_{2}, z_{3}\right\}$, is partitioned into $\mathcal{S}_{1}=\left\{z_{1}, z_{2}\right\}$ and $\mathcal{S}_{2}=\left\{z_{3}\right\}$. The matrix $W$ is now a $3 \times 3$ self-adjoint, positive matrix, and the block decomposition of $e^{-i W T}$ yields a $2 \times 2$ matrix $P$, a 2-dimensional column vector $Q=\left(Q_{1}, Q_{2}\right)^{T}$, a 2-dimensional row vector $R=\left(R_{1}, R_{2}\right)$ and a scalar $S$.

Fermi statistics The Fock space is $\mathfrak{F}=\mathbb{C}^{2} \otimes \mathbb{C}^{2} \otimes \mathbb{C}^{2}$. Given operators $a_{1}$ and $a_{2}$ at $t=0$, we seek an operator $b$ such that

$$
b=e^{-i \lambda T\left(a_{1}^{\dagger} a_{1}+a_{2}^{\dagger} a_{2}+b^{\dagger} b\right)}\left(R_{1} a_{1}+R_{2} a_{2}+S b\right) .
$$

Using the results above, the unique solution to this equation is

$$
b=\left(e^{i \lambda T a_{k}^{\dagger} a_{k}}-S\right)^{-1}\left(R_{1} a_{1}+R_{2} a_{2}\right),
$$

as may easily be checked by decomposing $\mathfrak{F}=\mathfrak{F}_{0} \oplus \mathfrak{F}_{1} \oplus \mathfrak{F}_{2}$ with $\mathfrak{F}_{r}$ the eigenspace of $a_{k}^{\dagger} a_{k}$ with eigenvalue $r$.

Substituting, we find that

$$
\left(\begin{array}{l}
a_{1}(T) \\
a_{2}(T)
\end{array}\right)=e^{-i \lambda T\left(a_{k}^{\dagger} a_{k}+b^{\dagger} b\right)}\left[P+Q\left(e^{i \lambda T a_{k}^{\dagger} a_{k}}-S\right)^{-1} R\right]\left(\begin{array}{l}
a_{1} \\
a_{2}
\end{array}\right) .
$$

In Appendix Q, we show that

$$
\left\langle 0\left|\left(a_{2}(T) a_{1}(T)+a_{1}(T) a_{2}(T)\right) a_{1}^{\dagger} a_{2}^{\dagger}\right| 0\right\rangle=F\left(\left|Q_{2}\right|^{2}-\left|Q_{1}\right|^{2}\right) \operatorname{det} P,
$$

and

$$
\left\langle 0\left|a_{1}(T)^{2} a_{1}^{\dagger} a_{2}^{\dagger}\right| 0\right\rangle=F Q_{1} \overline{Q_{2}} \operatorname{det} P
$$

where

$$
F=e^{-i \lambda T}\left\{\frac{1}{\bar{S}}\left[\frac{1}{e^{i \lambda T}-S}-\frac{e^{-i \lambda \alpha(S) T}}{1-S}\right]+\frac{e^{-i \lambda \alpha(S) T}-1}{1-|S|^{2}}\right\},
$$

and $\alpha(S)$ is defined by

$$
\alpha(S)=\frac{\left|R_{1}\right|^{2}+\left|R_{2}\right|^{2}}{|1-S|^{2}}=\frac{1-|S|^{2}}{|1-S|^{2}}
$$

Thus, except in the free case or for very carefully tuned parameters the CAR's are necessarily violated and the evolution is therefore nonunitary. Note that the coefficients of $F$ in 
Eqs. (5.21) and (5.22) vanish simultaneously for all $T$ if and only if $W$ is block diagonal with respect to the decomposition $\mathfrak{H}=\mathfrak{H}_{1} \oplus \mathfrak{H}_{2}$ (in which case $|S|=1$ and we are no longer in the generic case).

In the next section, it will be useful to have an explicit matrix representation for this evolution. Choosing a basis for the 8-dimensional Fock space $\mathfrak{F}$ such that

$$
a_{1}(0)=\left(\begin{array}{ccc}
0 & 1 & 0 \\
& 0 & 1 \\
0 & 0
\end{array}\right) \otimes \mathbb{1}_{2}, \quad a_{2}(0)=\left(\begin{array}{ccc}
0 & 1 & 0 \\
0 & 0 & 0 \\
0 & 0 & -1 \\
0 & 0
\end{array}\right) \otimes \mathbb{1}_{2}
$$

where $\mathbb{1}_{2}$ is the $2 \times 2$ identity matrix, one may show that

$$
a_{i}(T)=\left(\begin{array}{cccc}
0 & M_{i 2} & M_{i 1} & 0 \\
& 0 & N_{i 1} \\
& & & -N_{i 2} \\
& & & 0
\end{array}\right) \otimes \mathbb{1}_{2}
$$

Here $M_{i j}$ is the unitary matrix $M=P+Q(1-S)^{-1} R$ and $N$ is also a $2 \times 2$ unitary matrix given by $N=\left(P+Q\left(e^{i \lambda T}-S\right)^{-1} R\right) U$, where $U$ is another $2 \times 2$ unitary defined by

$$
\left.U_{i j} a_{j}\right|_{\mathfrak{F}_{2}}=\left.e^{-i \lambda T\left(a_{k}^{\dagger} a_{k}+b^{\dagger} b\right)} a_{i}\right|_{\mathfrak{F}_{2}}
$$

(which makes sense because the exponential preserves $\mathfrak{F}_{1}$ ). The precise form of $N$ will not concern us; however, we note that $M \neq N$, because $a_{1}(T)$ and $a_{2}(T)$ fail to anticommute. Bose statistics The Fock space is $\ell^{2} \otimes \ell^{2} \otimes \ell^{2}$ and the unique solution to Eq. (5.1) is

$$
b=f\left(d^{\dagger} d, c^{\dagger} c\right) c
$$

where

$$
c=\|R\|^{-1}\left(R_{1} a_{1}+R_{2} a_{2}\right), \quad d=\|R\|^{-1}\left(R_{2} a_{1}-R_{1} a_{2}\right),
$$

and $f(m, n)$ satisfies

$$
f(m, n+1)=\left(e^{i \lambda T\left[m+(n+1)\left(1+|f(m, n)|^{2}\right)\right]}-S\right)^{-1}\|R\|
$$

with $f(m, 0)=\left(e^{i \lambda T m}-S\right)^{-1}\|R\|$. Substituting back to determine $a_{i}(T)$, we show in Appendix $\triangle$ that

$$
\left\langle 0\left|\left(a_{2}(T) a_{1}(T)-a_{1}(T) a_{2}(T)\right) d^{\dagger} c^{\dagger}\right| 0\right\rangle=F\left(\left|Q_{1}\right|^{2}+\left|Q_{2}\right|^{2}\right) \operatorname{det} P,
$$

with $F$ given by Eq. (5.23). This should be compared with Eq. (5.21). Thus the evolution fails to be unitary on $\mathfrak{F}_{2}$. 


\section{DISCUSSION OF THE NONUNITARY EVOLUTION}

In the previous section, we showed that Model 2 was subject to a nonunitary evolution for both Bose and Fermi statistics. In this section, we discuss this evolution in more depth in the fermionic case. Recall that the Fock space $\mathfrak{F}$ is 8 -dimensional, and that the operators $\Psi_{\alpha}\left(0^{-}\right)(\alpha=1,2,3)$ represent the CAR's for three degrees of freedom on $\mathfrak{F}$. Writing $\Psi_{1, i}$ for the operators associated with points in $\mathcal{S}_{1}$, and $\Psi_{2}$ for the operator associated with the single element of $\mathcal{S}_{2}$, we write $\Psi_{1, i}\left(0^{-}\right)=a_{i}$ for $i=1,2$. The Heisenberg evolution $\Psi_{\alpha}\left(0^{-}\right) \rightarrow \Psi_{\alpha}\left(T^{+}\right)$is such that $\Psi_{1, i}\left(T^{+}\right)=a_{i}(T)$ and $\Psi_{2}\left(T^{+}\right)=\Psi_{2}\left(T^{-}\right)$. Our principal results in this section are, firstly, that the Heisenberg picture evolution cannot be expressed in either of the forms

$$
\Psi_{\alpha}\left(T^{+}\right)=X^{-1} \Psi_{\alpha}\left(0^{-}\right) X
$$

or

$$
\Psi_{\alpha}\left(T^{+}\right)=X^{\dagger} \Psi_{\alpha}\left(0^{-}\right) X
$$

for some operator $X$ on $\mathfrak{F}$; secondly, that the Heisenberg picture evolution does not admit an equivalent Schrödinger picture description in terms of a superscattering operator. In addition, we will discuss the problem of extending the evolution from that of the $\Psi_{\alpha}\left(0^{-}\right)$to arbitrary operators on $\mathfrak{F}$.

Firstly, then, we show that the Heisenberg picture evolution cannot be expressed in either of the forms Eq. (6.1) or (6.2). The form Eq. (6.1) is clearly impossible because it would entail $\left\{a_{1}(T), a_{2}(T)\right\}=0$, and we may dispose of Eq. (6.2) as follows. The explicit form of the $a_{i}(T)$ given above shows that any such operator $X$ would necessarily preserve the subspaces $\mathfrak{F}_{0}, \mathfrak{F}_{1}$ and $\mathfrak{F}_{2}$ of $\mathfrak{F}$; moreover, because

$$
\left.a_{i}(T)\right|_{\mathfrak{F}_{1}}=\left.M_{i j} a_{j}\right|_{\mathfrak{F}_{1}},
$$

where $M$ is unitary, we conclude that $\left.X\right|_{\mathfrak{F}_{1}}$ is unitary up to scale. Then it suffices to note that

$$
\begin{aligned}
\left\{a_{1}(T), a_{2}(T)\right\}|11\rangle= & X^{\dagger}\left(a_{1} X X^{\dagger} a_{2}\right. \\
& \left.+a_{2} X X^{\dagger} a_{1}\right) X|11\rangle
\end{aligned}
$$

which vanishes because $X$ preserves $\mathfrak{F}_{2}$ and $\left.X\right|_{\mathfrak{F}_{1}}$ is unitary up to scale. Accordingly, we cannot cast the evolution into either of the special forms Eq. (6.1) or (6.2).

Secondly, we show that the Heisenberg picture evolution cannot be described by a superscattering operator. Recall that a superscattering operator on the state space of a (separable) Hilbert space $\mathfrak{F}$ is a linear mapping $\$$ of the trace class operators $\mathcal{T}(\mathfrak{F})$ on $\mathfrak{F}$ such that if $\rho \in \mathcal{T}(\mathfrak{F})$ is a positive operator $\square^{\circ}$ of unit trace, then $\$ \rho$ is also a positive element of $\mathcal{T}(\mathfrak{F})$ with unit trace. Thus, $\$$ is a linear mapping of density matrices to density matrices, which need not preserve purity. If a superscattering operator $\$$ describes the Schrödinger picture

\footnotetext{
${ }^{6}$ For our purposes, a "positive operator" means one which is non-negative definite.
} 
evolution of a system, then the Heisenberg picture evolution is given by the linear mapping $\$^{\prime}$ of the bounded operators $\mathfrak{L}(\mathfrak{F})$ on $\mathfrak{F}$, defined by

$$
\operatorname{Tr} \rho\left(\$^{\prime} Z\right)=\operatorname{Tr}(\$ \rho) Z,
$$

for all $\rho \in \mathcal{T}(\mathfrak{F})$ and $Z \in \mathfrak{L}(\mathfrak{F})$. In fact, $\$^{\prime}$ is the dual mapping to $\$$ under the natural identification of $\mathfrak{L}(\mathfrak{F})$ with the dual space of $\mathcal{T}(\mathfrak{F})$.

The dual mapping $\$^{\prime}$ possesses three easily established properties: (i) $\$^{\prime} \mathbb{1}=\mathbb{1}$; (ii) $\left(\$^{\prime} Z\right)^{\dagger}=\$^{\prime}\left(Z^{\dagger}\right)$ for all $Z$; and (iii) $\$^{\prime}$ is positive in the sense that $\$^{\prime} Z$ is a positive operator whenever $Z$ is. If one writes the superscattering operator using the index notation (e.g., [18])

$$
(\$ \rho)^{A}{ }_{B}=\$_{B C}^{A}{ }^{D} \rho^{C}{ }_{D}
$$

then $\$^{\prime}$ may be written as

$$
\left(\$^{\prime} Z\right)_{C}^{D}=\$_{B C}^{A} Z_{A}^{B}
$$

Returning to our case of interest, we now show that there is no superscattering operator $\$$ for which the Heisenberg evolution can be written as

$$
\Psi_{\alpha}\left(T^{+}\right)=\$^{\prime} \Psi_{\alpha}\left(0^{-}\right)
$$

We will need the fact that if $u=\left(u_{1}, u_{2}\right)^{T}$ and $v=\left(v_{1}, v_{2}\right)^{T}$ are 2-dimensional complex column vectors and $\alpha \in \mathbb{R}$, then the eigenvalues $\mu$ of the matrix

$$
K=\left(\begin{array}{cccc}
\alpha & u_{2} & u_{1} & 0 \\
\overline{u_{2}} & \alpha & 0 & v_{1} \\
\overline{u_{1}} & 0 & \alpha & -v_{2} \\
0 & \overline{v_{1}} & -\overline{v_{2}} & \alpha
\end{array}\right) \otimes \mathbb{1}_{2}
$$

satisfy

$$
(\mu-\alpha)^{4}-(\mu-\alpha)^{2}\left(u^{\dagger} u+v^{\dagger} v\right)+\left|u^{\dagger} v\right|^{2}=0 .
$$

Note that the operator $C=\alpha \mathbb{1}+\beta a_{1}+\bar{\beta} a_{1}^{\dagger}+\gamma a_{2}+\bar{\gamma} a_{2}^{\dagger}$ takes the form (6.9) with $u=v=$ $(\beta, \gamma)^{T}$ and therefore has eigenvalues

$$
\mu=\alpha \pm \sqrt{|\beta|^{2}+|\gamma|^{2}}
$$

Accordingly, $C$ is positive if and only if $\alpha \geq \sqrt{|\beta|^{2}+|\gamma|^{2}}$. Now suppose that there exists a superscattering operator $\$$ such that Eq. (6.8) holds. Then using the properties (i) and (ii) of $\$^{\prime}$, we have

$$
\begin{aligned}
\$^{\prime} C= & \alpha \mathbb{1}+\beta a_{1}(T)+\bar{\beta} a_{1}(T)^{\dagger} \\
& +\gamma a_{2}(T)+\bar{\gamma} a_{2}(T)^{\dagger},
\end{aligned}
$$

which may be seen to take the form (6.9) with $u=((\beta, \gamma) M)^{T}, v=((\beta, \gamma) N)^{T}$. Hence its eigenvalues are 


$$
\mu=\alpha \pm \sqrt{|\beta|^{2}+|\gamma|^{2} \pm \Delta}
$$

where the two \pm signs are independent and

$$
\Delta=\sqrt{\left(|\beta|^{2}+|\gamma|^{2}\right)^{2}-\left|u^{\dagger} v\right|^{2}}
$$

is real and positive by the Schwarz inequality and the unitarity of $M$ and $N$. Because these matrices are unequal, we may choose $\beta$ and $\gamma$ so that $\Delta>0$. Choosing $\alpha$ to lie in the range

$$
\sqrt{|\beta|^{2}+|\gamma|^{2}}<\alpha<\sqrt{|\beta|^{2}+|\gamma|^{2}+\Delta}
$$

the operator $C$ is positive, but $\$^{\prime} C$ is not. Accordingly, $\$^{\prime}$ violates property (iii) above and therefore cannot be the dual of a superscattering operator.

Next, we consider the Heisenberg evolution itself in more detail. It is worth pointing out that we have not by any means obtained the full Heisenberg picture evolution; at present we know the evolution of only a 3-dimensional subspace (spanned by the $\Psi_{\alpha}\left(0^{-}\right)$) of the 64-dimensional space $\mathfrak{L}(\mathfrak{F})$ of linear operators on the 8-dimensional Hilbert space $\mathfrak{F}$. Owing to our results above, various natural strategies for extending this evolution to the whole of $\mathfrak{L}(\mathfrak{F})$ are denied to us: the evolution cannot be extended as a $*$-homomorphism (i.e., mapping any polynomial in the $\Psi_{\alpha}\left(0^{-}\right)$to the corresponding polynomial in the $\left.\Psi_{\alpha}\left(T^{+}\right)\right)$ because the CAR's are violated; we cannot write $Z \rightarrow X^{-1} Z X$ or $Z \rightarrow X^{\dagger} Z X$ because of our observations above, nor can we write $Z \rightarrow \$^{\prime} Z$ for some superscattering operator $\$$.

It therefore seems that there is no natural extension of our evolution to $\mathfrak{L}(\mathfrak{F})$. As a concrete illustration of this type of behaviour, let us consider an example with one fermionic degree of freedom. Define the operator $a$ on $\mathbb{C}^{2}$ by

$$
a=\left(\begin{array}{ll}
0 & 1 \\
0 & 0
\end{array}\right)
$$

and suppose an evolution is given such that $\mathbb{1} \rightarrow \mathbb{1}, a \rightarrow \mu a$ and $a^{\dagger} \rightarrow \mu a^{\dagger}$, where $0 \leq \mu<1$. It turns out that there are at least two choices for the evolution of $a^{\dagger} a$ consistent with a superscattering operator description. The first is that $a^{\dagger} a \rightarrow a^{\dagger} a$, corresponding to a superscattering operator $\$$ with action

$$
\$\left(\begin{array}{cc}
\alpha & \beta \\
\bar{\beta} & 1-\alpha
\end{array}\right)=\left(\begin{array}{cc}
\alpha & \mu \beta \\
\mu \bar{\beta} & 1-\alpha
\end{array}\right),
$$

on the state space of $\mathbb{C}^{2}$, whilst the second is $a^{\dagger} a \rightarrow a a^{\dagger}$ and corresponds to the superscattering operator $£$ with action

$$
£\left(\begin{array}{cc}
\alpha & \beta \\
\bar{\beta} & 1-\alpha
\end{array}\right)=\left(\begin{array}{cc}
1-\alpha & \mu \beta \\
\mu \bar{\beta} & \alpha
\end{array}\right) .
$$

To conclude this section, we note that the failure of positivity which showed the nonexistence of a superscattering operator can be traded for a loss of the trace preserving property: by allowing $\mathbb{1} \rightarrow \kappa \mathbb{1}$ for $\kappa \geq \sqrt{2}$, any positive $C$ of the form discussed above is mapped to a positive operator. One might therefore attempt to extend this in some way to a positive evolution on the whole of $\mathfrak{L}(\mathfrak{F})$ (which can be done if the evolution on $\mathbb{1}, a_{i}(0), a_{i}(0)^{\dagger}$ is 
completely positive - see Theorem 1.2.3 in Arveson [29]) thereby obtaining (by duality) a Schrödinger picture evolution possessing all the properties of a superscattering matrix except the preservation of trace. Rather than allowing individual probabilities to be negative with total probability equal to unity, we would now have positive probabilities with a total in excess of unity. It would be tempting to rescale this total to remove this problem, but that would amount to rescaling $a_{i}(T)$, for which there is no obvious justification.

\section{THE CLASSICAL LIMIT}

With the normal ordering used above, we have shown that the quantum theory is uniquely determined in the generic case for all values of the coupling constant $\lambda$. On the other hand, we have also seen that the classical theory is nonunique in the strong coupling regime. It is therefore interesting to determine the extent to which the classical theory may be regarded as a limit of the quantum theory.

We consider Model 1 with Bose statistics. Reintroducing the units of action by replacing $a$ and $b$ by $\hbar^{1 / 2} a$ and $\hbar^{1 / 2} b$ respectively, the consistency requirement Eq. (5.1) becomes

$$
b=e^{-i \hbar \lambda T\left(a^{\dagger} a+b^{\dagger} b\right)}(R a+S b),
$$

in which $a$ and $a^{\dagger}$ obey the CCR's $\left[a, a^{\dagger}\right]=1$. The unique solution to this is $b=f\left(\hbar \lambda T ; a^{\dagger} a\right) a$, where

$$
f(\nu ; n+1)=\left(e^{i \nu(n+1)\left(1+|f(\nu ; n)|^{2}\right)}-S\right)^{-1} R,
$$

with $f(\nu ; 0)=(1-S)^{-1} R$ for all $\nu$.

One expects the classical limit to be

$$
b_{\mathrm{cl}}=g\left(\lambda T\left|a_{\mathrm{cl}}\right|^{2}\right) a_{\mathrm{cl}},
$$

where

$$
g(\mu)=\lim _{n \rightarrow \infty} f(\mu / n ; n),
$$

which implements the limit $\hbar \rightarrow 0$ while keeping $\lambda T \hbar n=\mu$ fixed, where we define $\mu=$ $\lambda T\left|a_{\mathrm{cl}}\right|^{2}$. Note that the classical quantities $a_{\mathrm{cl}}$ and $b_{\mathrm{cl}}$ have dimensions of (action) $)^{1 / 2}$. If the limit $g(\mu)$ exists uniformly in $\mu$ in some neighbourhood, then we have

$$
b_{\mathrm{cl}}=\left(e^{i \lambda T\left(\left|a_{\mathrm{cl}}\right|^{2}+\left|b_{\mathrm{cl}}\right|^{2}\right)}-S\right)^{-1} R,
$$

for initial data corresponding to this neighbourhood. This is, of course, equivalent to the classical consistency condition Eq. (3.8) for this model.

Thus it is important to clarify the convergence properties of $g_{n}(\mu)=f(\mu / n ; n)$ as $n \rightarrow \infty$. This turns out to be somewhat delicate and we have not as yet brought the general case fully under analytic control. However, we have investigated the problem numerically in the particular example $P=-Q=R=S=1 / \sqrt{2}$ and $A=B=1$ for various values of $\mu$. With these parameters, it is easy to see that $\left(g_{n}(\mu)^{-1}+1\right) / \sqrt{2}$ lies on the unit circle for all $n, \mu$; accordingly we define 


$$
\theta_{n}=\arg \frac{g_{n}(\mu)^{-1}+1}{\sqrt{2}},
$$

where $-\pi<\arg z \leq \pi$. In Table 【, we give the values of $\theta_{n}$ for $n=500,1000,1500,2000$ and 2500 with $\mu=0.5,3.0,7.0$, and 13.0 and also give the value of $\theta$ corresponding to the nearest solution to the classical equation of motion. These results suggest a slow convergence to a unique classical limit for each of these values of $\mu$. At these values of $\mu$, the classical system has $1,7,13$ and 25 solutions respectively (see Figures 1 and 2 for the cases $\mu=0.5$ and $\mu=3.0$ ) of which the quantum theory picks out precisely one. However, it is by no means the case that $\theta_{n}$ converges for any value of $\mu$. Indeed, it appears that convergence occurs only for certain bands of $\mu$, the first two of which are $0 \leq \mu \lesssim 3.5$ and $6.7 \lesssim \mu \lesssim 8.5$. We believe that this band structure continues indefinitely; it seems reasonable to assume that the bands become narrower as $\mu$ increases.

One may interpret these results as indicating that for those ranges of the coupling strength $\mu=\lambda T\left|a_{\mathrm{cl}}\right|^{2}$ in which the classical limit exists, the unique classical solution isolated by the quantum theory is the correct classical description of the dynamics. However, for other values of the coupling strength there is no classical limit and presumably no valid classical description of the dynamics. Finally, we note that these results are strongly dependent on the choice of operator ordering as we will see in the next section.

\section{OPERATOR ORDERING}

So far, we have worked with a single choice of operator ordering, namely the literal ordering of Eq. (2.2) which corresponds to normal ordering of the quantised Hamiltonian. In this section, we briefly discuss the effect of allowing alternative orderings in which Eq. (2.2) is ordered as

$$
\dot{\psi}=-i W \psi-i \alpha \lambda\left(\psi^{\dagger} \psi\right) \psi-i(1-\alpha) \lambda \psi\left(\psi^{\dagger} \psi\right)
$$

for $\alpha \in[0,1]$. The foregoing treatment is the case $\alpha=1$.

Consider Model 1 for Bose statistics. The analogue of Eq. (5.1) is

$$
b=e^{-i \alpha \lambda T\left(a^{\dagger} a+b^{\dagger} b\right)}(R a+S b) e^{-i(1-\alpha) \lambda T\left(a^{\dagger} a+b^{\dagger} b\right)},
$$

(we have set $A=\mathbb{1}$ for simplicity). Making the ansatz $b=f\left(a^{\dagger} a\right) a$, we find that $f$ satisfies

$$
f(0)=(R+S f(0)) e^{-i \lambda T(1-\alpha)\left(1+|f(0)|^{2}\right)},
$$

and

$$
\begin{aligned}
f(n+1)= & e^{-i \lambda T(n+1) \alpha\left(1+|f(n)|^{2}\right)}(R+S f(n+1)) \\
& \times e^{-i \lambda T(n+2)(1-\alpha)\left(1+|f(n+1)|^{2}\right)},
\end{aligned}
$$

for $n \geq 0$. The case $\alpha=1$ was treated in Section $\square$ and uniquely determines $f(n+1)$ in terms of $f(n)$ for each $n$. However, the case $\alpha=0$ is rather different and is described by

$$
f(n)=e^{-i \lambda T \hbar(n+1)\left(1+|f(n)|^{2}\right)}(R+S f(n)),
$$


where we have written $\hbar$ explicitly. It is easy to recast this into the form of the classical consistency requirement Eq. (3.8) and it follows that $f(n)$ is uniquely determined for small quantum numbers $n \hbar \ll(\lambda T)^{-1}$ but not for $n \hbar \gg(\lambda T)^{-1}$, i.e., classical nonuniqueness reemerges at high quantum numbers. There are therefore many functions $f(n)$ solving Eq. (8.5), each one of which corresponds to a different "branch" of the quantum theory. Most of these branches do not possess a classical limit. However, in contrast to the situation for normal ordering, every classical solution will arise as the classical limit of some branch of the quantum theory.

It would be interesting if the nonunitarity of Model 2 could be removed by a suitable ordering prescription. In Appendix Q, we investigate this for orderings of form (8.1) with the ansatz $b=f\left(d^{\dagger} d, c^{\dagger} c\right) c$ with $c$ and $d$ given by Eq. (5.29). (Solutions do exist which take this form.) For all $0 \leq \alpha \leq 1$, we find that the (anti)commutation relations are violated for generic values of the parameters.

\section{SELF-CONSISTENT PATH INTEGRAL}

\section{A. General Formalism}

In this section, we compare the results obtained from the QIVP with those obtained using the self-consistent path integral developed by Thorne and collaborators [4, [0, 30] and employed by Politzer [1]. To establish our notation, we briefly review the quantisation of our system by path integral methods in the absence of CTC's. Starting with the bosonic case, it is convenient to use the holomorphic representation (see, e.g., [31]) in which the Hilbert space $\mathfrak{F}$ is the space of analytic functions $f\left(\overline{c_{1}}, \ldots, \overline{c_{s}}\right)$ with inner product

$$
\langle f \mid g\rangle=\int \mathcal{D} c^{\dagger} \mathcal{D} c e^{-c^{\dagger} c} \overline{f\left(c^{\dagger}\right)} g\left(c^{\dagger}\right)
$$

where we write $c^{\dagger}$ to denote $\left(\overline{c_{1}}, \ldots, \overline{c_{s}}\right)$ and the measure is

$$
\mathcal{D} c^{\dagger} \mathcal{D} c=\prod_{j} \frac{d \overline{c_{j}} d c_{j}}{2 \pi i}
$$

The Hilbert space $\mathfrak{F}$ carries a (Fock) representation of the CCR's in which $c_{j}^{\dagger}$ acts as multiplication by $\overline{c_{j}}$ and $c_{j}$ as $\partial / \partial \overline{c_{j}}$. Operators on $\mathfrak{F}$ are described by their kernels:

$$
(A f)\left(c^{\dagger}\right)=\int \mathcal{D} c^{\prime \dagger} \mathcal{D} c^{\prime} e^{-c^{\prime \dagger} c^{\prime}} A\left(c^{\dagger} ; c^{\prime}\right) f\left(c^{\prime \dagger}\right)
$$

In particular, if $K$ is a $s \times s$ matrix, then the mapping $f\left(c^{\dagger}\right) \rightarrow f\left(c^{\dagger} K\right)$ has kernel $\exp c^{\dagger} K c^{\prime}$.

Starting with the (normal ordered) quantised bosonic Hamiltonian $H$ on $\mathfrak{F}$, one may obtain the kernel for $U=e^{-i H t}$ in the form

$$
U_{t}\left(c^{\dagger} ; c^{\prime}\right)=\int \prod_{t^{\prime}} \mathcal{D} \gamma\left(t^{\prime}\right)^{\dagger} \mathcal{D} \gamma\left(t^{\prime}\right) \exp \left\{\frac{1}{2}\left(\gamma^{\dagger}(t) \gamma(t)+\gamma^{\dagger}(0) \gamma(0)\right)+i S[\gamma]\right\}
$$

where the action functional $S[\gamma]$ is defined in terms of the classical Hamiltonian (2.6) by 


$$
S[\gamma]=\int_{0}^{t}\left(\frac{i}{2}\left(\gamma\left(t^{\prime}\right)^{\dagger} \dot{\gamma}\left(t^{\prime}\right)-\dot{\gamma}\left(t^{\prime}\right)^{\dagger} \gamma\left(t^{\prime}\right)\right)-H\left(\gamma\left(t^{\prime}\right), i \gamma\left(t^{\prime}\right)^{\dagger}\right)\right) d t^{\prime}
$$

and the paths $\gamma\left(t^{\prime}\right)$ are subject to the boundary conditions $\gamma^{\dagger}(t)=c^{\dagger}$ and $\gamma(0)=c^{\prime}$. In the free case, for example, one may evaluate the path integral explicitly to give

$$
U_{t}\left(c^{\dagger} ; c^{\prime}\right)=\exp c^{\dagger} e^{-i W t} c^{\prime}
$$

One may develop the path integral treatment for Fermi statistics in a parallel fashion 31 by replacing the integration variables by Grassmann numbers and $\mathcal{D} c \mathcal{D} c^{\dagger}$ by Berezin measure. Again, the resulting kernel has the action of $e^{-i H t}$ on $\mathfrak{F}$, where $H$ is now the fermionic normal ordered quantised Hamiltonian.

A natural generalisation of this to enable the treatment of chronology violating systems is the self-consistent path integral [1, 4, 7, 30]. Instead of integrating over all field configurations with $\gamma(0)=c^{\prime}$ and $\gamma^{\dagger}(T)=c^{\dagger}$ to form the kernel $U_{T}\left(c^{\dagger}, c^{\prime}\right)$, the self-consistent path integral prescription requires that one should restrict the class of field configurations to those obeying the self-consistency requirements imposed by any CTC's present (here, the boundary conditions (2.7)). To implement this, we first decompose $\mathfrak{F}=\mathfrak{F}_{1} \otimes \mathfrak{F}_{2}$, where $\mathfrak{F}_{1}$ is the space of analytic functions in variables $\overline{a_{1}}, \ldots, \overline{a_{s_{1}}}$, and $\mathfrak{F}_{2}$ is the space of analytic functions in $\overline{b_{1}}, \ldots, \overline{b_{s_{2}}}$. The (self-consistent) evolution kernel from $t=0^{-}$to $t=T^{+}$can then be written in the form

$$
X\left(a^{\dagger}, b^{\dagger} ; a^{\prime}, b^{\prime}\right)=\mathcal{N} e^{b^{\dagger} B b^{\prime}} \widetilde{U_{T}}\left(a^{\dagger} ; a^{\prime}\right)
$$

Here, $\mathcal{N}$ is a normalisation constant and the factor $e^{b^{\dagger} B b^{\prime}}$ implements the boundary condition $\psi_{2}\left(T^{+}\right)=B \psi_{2}\left(0^{-}\right)$while $\widetilde{U_{T}}$ is given by the same path integral as $U_{T}$ but taken over all field configurations with $\gamma(0)=\left(a^{\prime}, b^{\prime \prime}\right), \gamma^{\dagger}(T)=\left(a, A b^{\prime \prime}\right)^{\dagger}$ for any $b^{\prime \prime}$. As noted by Politzer [1], $\widetilde{U_{T}}\left(a^{\dagger} ; a^{\prime}\right)$ may be obtained from $U_{T}\left(a^{\dagger}, b^{\dagger} ; a^{\prime}, b^{\prime}\right)$ by setting $b=A b^{\prime}$ and integrating over all possibilities in the Hilbert space measure of $\mathfrak{F}_{2}$, that is,

$$
\widetilde{U_{T}}\left(a^{\dagger} ; a^{\prime}\right)=\int \mathcal{D} b^{\dagger} \mathcal{D} b e^{-b^{\dagger} b} U_{T}\left(a^{\dagger}, b^{\dagger} A^{\dagger} ; a^{\prime}, b\right),
$$

which may be rewritten in the form

$$
\begin{aligned}
\widetilde{U_{T}}\left(a^{\dagger} ; a^{\prime}\right)= & \int \mathcal{D} b^{\dagger} \mathcal{D} b \int \mathcal{D} c^{\dagger} \mathcal{D} c e^{-b^{\dagger} b-c^{\dagger} c} \\
& \times e^{b^{\dagger} A^{\dagger} c} U_{T}\left(a^{\dagger}, c^{\dagger} ; a^{\prime}, b\right) .
\end{aligned}
$$

Thus, by expanding $e^{b^{\dagger} A^{\dagger} c}$ as

$$
e^{b^{\dagger} A^{\dagger} c}=\prod_{i=1}^{s_{2}} \sum_{n_{i}=0}^{\infty} \frac{\left(A^{\dagger} c\right)_{i}^{n_{i}}\left(b_{i}^{\dagger}\right)^{n_{i}}}{n_{i} !}
$$

we obtain the matrix element $\left\langle\mathbf{m}\left|\widetilde{U_{T}}\right| \mathbf{m}^{\prime}\right\rangle$ in the form

$$
\left\langle\mathbf{m}\left|\widetilde{U_{T}}\right| \mathbf{m}^{\prime}\right\rangle=\sum_{\mathbf{n}}\left\langle\mathbf{m} ; \tilde{\mathbf{n}}\left|U_{T}\right| \mathbf{m}^{\prime} ; \mathbf{n}\right\rangle,
$$


where the vector $|\mathbf{m}\rangle \in \mathfrak{F}_{1}$ is the function $\prod_{i}\left(m_{i} !\right)^{-1 / 2}{\overline{a_{i}}}^{m_{i}}$, and the vector $|\tilde{\mathbf{n}}\rangle \in \mathfrak{F}_{2}$ is the function $\prod_{i}\left(n_{i} !\right)^{-1 / 2}{\overline{\left(A^{\dagger} b\right)_{i}}}_{i}$. In addition, $\left(A^{\dagger} b\right)_{i}$ denotes $\sum_{j} A_{i j}^{\dagger} b_{j}$. We refer to Eq. (9.11), which is a generalisation of the expression given by Politzer [i] as the partial trace definition of the self-consistent path integral.

The fermionic case follows a similar pattern, when one replaces the integration variables by Grassmann numbers and uses Berezin measure; the main difference lies in the partial trace definition. Starting from the analogue of (9.9), we expand $e^{b^{\dagger} A^{\dagger} c}$ as

$$
\begin{aligned}
e^{b^{\dagger} A^{\dagger} c} & =\prod_{i=1}^{s_{2}}\left(1+b_{i}^{\dagger}\left(A^{\dagger} c\right)_{i}\right) \\
& =\sum_{\mathbf{n}}(-1)^{n} \prod_{i}\left(A^{\dagger} c\right)_{i}^{n_{i}}\left(b_{i}^{\dagger}\right)^{n_{i}}
\end{aligned}
$$

where $n=\sum_{i} n_{i}$, and therefore obtain

$$
\left\langle\mathbf{m}\left|\widetilde{U_{T}}\right| \mathbf{m}^{\prime}\right\rangle=\sum_{\mathbf{n}}(-1)^{n}\left\langle\mathbf{m} ; \tilde{\mathbf{n}}\left|U_{T}\right| \mathbf{m}^{\prime} ; \mathbf{n}\right\rangle
$$

under the assumption that the Grassmann number $b_{i}^{\dagger}$ commutes with the kernel of $U_{T}\left(a^{\dagger}, c^{\dagger} ; a^{\prime}, b\right)$, which holds if $H$ conserves particle number (as it does in our case of interest). The factor of $(-1)^{n}$ was omitted by Politzer [1]; it arises because terms of the form

$b_{i}^{\dagger}\left(A^{\dagger} c\right)_{i}$ coming from $e^{b^{\dagger} A^{\dagger} c}$ must be rearranged in order to move the $b_{i}^{\dagger}$ 's into the ket and the $\left(A^{\dagger} c\right)_{i}$ 's into the bra of the matrix element $\left\langle\mathbf{m} ; \tilde{\mathbf{n}}\left|U_{T}\right| \mathbf{m}^{\prime} ; \mathbf{n}\right\rangle$. In Appendix $\mathbb{B}$, we will see how, for free fields, these factors ensure that the evolution computed from (9.13) agrees with that obtained directly from the path integral, and also with that obtained from the QIVP.

\section{B. Free Fields}

Whilst one can use the partial trace definition to compute the quantum evolution $X$ for free fields (see Appendix B), it is easier to evaluate the path integral directly, using the fact that the kernel of the free evolution is given by

$$
U_{T}\left(c^{\dagger} ; c^{\prime}\right)=\exp c^{\dagger} e^{-i W T} c^{\prime}
$$

Writing $e^{-i W T}$ in the block form (3.1) as above, we obtain

$$
\begin{aligned}
\widetilde{U_{T}}\left(a^{\dagger} ; a^{\prime}\right)= & \int \mathcal{D} b^{\dagger} \mathcal{D} b \exp \left\{-b^{\dagger}\left(\mathbb{1}-A^{\dagger} S\right) b+a^{\dagger} P a^{\prime}\right. \\
& \left.+a^{\dagger} Q b+b^{\dagger} A^{\dagger} R a^{\prime}\right\}
\end{aligned}
$$

which may be evaluated to give

$$
\widetilde{U_{T}}\left(a^{\dagger} ; a^{\prime}\right)=\left(\operatorname{det}\left(\mathbb{1}-A^{\dagger} S\right)\right)^{-1} \exp a^{\dagger} M a^{\prime},
$$

where $M=\left(P+Q(A-S)^{-1} R\right)$. In the generic case, the convergence of the path integral is guaranteed because $\left\|A^{\dagger} S\right\|<1$ and so $\mathbb{1}-A^{\dagger} S$ has positive hermitian part. 
Noting that $V\left(a^{\dagger} ; a^{\prime}\right)=\exp a^{\dagger} M a^{\prime}$ is the unitary kernel, because $M$ is unitary, we conclude that the unitary kernel obtained from the self-consistent path integral is

$$
X\left(a^{\dagger}, b^{\dagger} ; a^{\prime}, b^{\prime}\right)=\exp \left\{a^{\dagger} M a^{\prime}+b^{\dagger} B b^{\prime}\right\}
$$

whose corresponding operator $X$ acts on annihilation operators $a_{i}$ and $b_{i}$ according to

$$
X^{\dagger} a_{i} X=M_{i j} a_{j}, \quad X^{\dagger} b_{i} X=B_{i j} b_{j}
$$

Moreover, the normalisation constant is given by $\mathcal{N}=\operatorname{det}\left(\mathbb{1}-A^{\dagger} S\right)$.

In the fermionic case, the path integral may be evaluated explicitly to obtain a unitary evolution with the action (9.18) on annihilation operators and normalisation constant $\mathcal{N}=$ $\operatorname{det}\left(\mathbb{1}-A^{\dagger} S\right)^{-1}$.

Thus in both cases, we have obtained agreement with the QIVP evolution. Moreover, we have given a general proof of the unitarity of free field evolution using the self-consistent path integral; previously this had only been established in a particular case [1].

\section{An Interacting Model}

We study Model 1 of Section $\nabla$ for both Bose and Fermi statistics, employing the partial trace definition, and choosing the normalisation constant so that $\langle 0 ; 0|X| 0 ; 0\rangle=1$, which is reasonable because the Hamiltonian $H$ is particle-number preserving. In the fermionic case, we obtain

$$
\begin{aligned}
\left\langle 0\left|\widetilde{U_{T}}\right| 0\right\rangle & =\left\langle 00\left|e^{-i H T}\right| 00\right\rangle-\left\langle 01\left|e^{-i H T}\right| 01\right\rangle \\
& =1-S \\
\left\langle 1\left|\widetilde{U_{T}}\right| 1\right\rangle & =\left\langle 10\left|e^{-i H T}\right| 10\right\rangle-\left\langle 11\left|e^{-i H T}\right| 11\right\rangle \\
& =P-(P S-R Q) e^{-i \lambda T},
\end{aligned}
$$

from which it follows that the evolution from $t=0^{-}$to $t=T^{+}$is given by

$$
\langle m ; n|X| m ; n\rangle=\delta_{n n^{\prime}} \delta_{m m^{\prime}} f(m)
$$

where

$$
f(m)=\left\{\begin{array}{cc}
1 & m=0 \\
\frac{P-(P S-R Q) e^{-i \lambda T}}{1-S} & m=1 .
\end{array}\right.
$$

Thus $X$ is nonunitary in general, which is essentially the result obtained by Politzer [1] in special cases, modulo some changes of sign owing to the factors of $(-1)^{n}$ discussed above. Except when $\lambda T /(2 \pi) \in \mathbb{Z}$ this differs from the unitary evolution obtained from the QIVP.

In the bosonic case, we have

$$
\left\langle m\left|\widetilde{U_{T}}\right| m^{\prime}\right\rangle=\sum_{n=0}^{\infty} \frac{e^{-i \lambda T(m+n)(m+n-1) / 2}}{(m ! n !)^{1 / 2}}\left\langle 00\left|(R a+S b)^{n}(P a+Q b)^{m}\right| m^{\prime} n\right\rangle,
$$

and therefore conclude that $\left\langle m n|X| m^{\prime} n^{\prime}\right\rangle=\delta_{m m^{\prime}} \delta_{n n^{\prime}} f(m)$ with $f(0)=1$ and 


$$
f(m)=\frac{\sum_{n=0}^{\infty} e^{-i \lambda T(m+n)(m+n-1) / 2} \sum_{r=\max \{n-m, 0\}}^{n}\left(\begin{array}{c}
n \\
r
\end{array}\right)\left(\begin{array}{c}
m \\
n-r
\end{array}\right)(R Q)^{n-r} P^{m+r-n} S^{r}}{\sum_{n} S^{n} e^{-i \lambda T n(n-1) / 2}} .
$$

One may show that $X$ fails to be unitary in general. Again, it clearly differs from the unitary evolution obtained from the QIVP.

\section{CONCLUSION}

In this paper, we have analysed in detail the classical and quantum behaviour of a class of nonlinear chronology violating systems. Classically, we found that unique solutions exist for all choices of initial data in the linear and weak-coupling regimes, whilst the solutions become nonunique in the strong-coupling regime. This confirms the expectation that the behaviour of nonlinear fields interpolates between that of classical linear fields and hardsphere mechanics. Quantum mechanically, we have shown that one can make sense of the quantum initial value problem for chronology violating systems; moreover, (at least with a natural choice of operator ordering) the quantum dynamics is unique for all values of the coupling constant. We have also exhibited examples in which this evolution does not preserve the (anti)commutation relations; it seems highly likely that this is the general situation. Moreover, the nonunitary evolution cannot be described by a superscattering operator - the loss of unitarity is more radical than previously thought, e.g., by Hawking [18.

We have also compared our quantum evolution with that computed using the selfconsistent path integral, and found that they do not agree. This is not surprising, because the equivalence of these approaches for non-chronology violating systems relies on the existence of a foliation by Cauchy surfaces and there is no a priori reason to expect the equivalence to persist in the presence of CTC's. In this regard it is interesting that the QIVP and self-consistent path integral are nonetheless equivalent for linear fields. To some extent, it is a matter of taste which approach one prefers. We prefer the QIVP approach for two main reasons. Firstly, we have found circumstances (e.g., Model 1 in Section VC) in which one obtains a unitary theory from the QIVP but not from the path integral. Secondly, the effect of the CTC's in our models is to introduce constraints which lead to a nontrivial geometric structure in the classical phase space. We suspect that the quantisation of this system requires more than just a restriction of the class of allowed histories, and that the path integral measure should also be modified (a similar comment has also been made in [16]). A hint of this appears in the treatment of linear fields, in which the propagator obtained from the self-consistent path integral must be rescaled by a factor of $\operatorname{det}\left(\mathbb{1}-A^{\dagger} S\right)^{ \pm 1}$. It is plausible that in the linear case, the required modification to the path integral measure reduces to rescaling by this constant factor, but that for the nonlinear case the modification is nontrivial. At present it is not clear to us exactly how the path integral should be modified; on the other hand it is clear that the QIVP does correctly implement the CTC constraints and remains close to the spirit of the classical treatment.

The relationship between the unique quantum theory and the nonunique classical theory is intriguing. We have seen that there exist ranges of the coupling strength in which the quantum theory has a classical limit which selects precisely one of the many classical 
solutions, and other ranges in which no classical limit exists. It would be interesting to understand the underlying reasons for this behaviour in more detail.

Finally, it is curious that the classical symplectic structure can be preserved for systems which do not preserve the quantum commutation relations. It is tempting to wonder whether there is a way of quantising these models so that unitarity is preserved. Our uniqueness result for the QIVP rules this out within a Hilbert space context (at least with normal operator ordering) but it is possible that the situation might be different for the QIVP on an indefinite (Krein) inner product space in which irreducible non-Fock representations of the CCR's exist for even a single degree of freedom [32]. The motivation for studying Krein spaces would be that the loss of physical degrees of freedom in the nonchronal region might be equivalent to the addition of unphysical states with negative norm-squared.

\section{ACKNOWLEDGMENTS}

We thank John Friedman for correspondence on the preservation of the symplectic structure and Simon Eveson, Petr Hájíček and Bernard Kay for useful and clarifying conversations. The work of CJF was supported by the Royal Society, the Schweizerischer Nationalfonds and EPSRC Grant No. GR/K 29937 to the University of York. The work of AH was supported in part by the Schweizerischer Nationalfonds and the Tomalla Foundation at the Universität Bern.

\section{APPENDIX A: PATH INTEGRAL APPROACH TO THE FREE CLASSICAL EVOLUTION}

In this Appendix, we show how the classical evolution derived in Section IIIA may be reproduced using a method due to Goldwirth et al. [6] and based on path integrals. (Goldwirth et al. regarded the classical wave equation as the first quantisation of an underlying particle mechanics.) The central idea is to sum the propagators for all possible trajectories through the CTC region. We will use this method to determine the propagator between $t=0^{-}$and $t=T^{+}$, essentially repeating the calculation of [6] in our (slightly simpler) notation.

The block matrix decomposition Eq. (3.1) suggests that we break the problem into four parts, evaluating the propagators from $\mathcal{S}_{i}$ at $t=0^{-}$to $\mathcal{S}_{j}$ at $t=T^{+}$separately for each $i, j=1,2$. Note that a particle on $\mathcal{S}_{2}$ at $t=0^{-}$must enter the wormhole there and reemerge on $\mathcal{S}_{2}$ at $t=T^{+}$. Thus the $\mathcal{S}_{2} \rightarrow \mathcal{S}_{2}$ propagator equals $B$, whilst that for $\mathcal{S}_{2} \rightarrow \mathcal{S}_{1}$ vanishes. In addition, the propagator $\mathcal{S}_{1} \rightarrow \mathcal{S}_{2}$ also vanishes by the time reverse of this argument. It remains to compute the propagator for $\mathcal{S}_{1} \rightarrow \mathcal{S}_{1}$. In this case, there are countably many possible trajectories. The particle can either go directly to $\mathcal{S}_{1}$ with propagator $P$, or it can enter the CTC region to arrive at $\mathcal{S}_{2}$ at $t=T^{-}$(propagator $R$ ), pass through the wormhole to $\mathcal{S}_{2}$ at $t=0^{+}$(propagator $A^{-1}$ ), execute $n$ circuits of the CTC's (propagator $\left(A^{-1} S\right)^{n}$ ) and finally travel from $\mathcal{S}_{2}$ at $t=0^{+}$to $\mathcal{S}_{1}$ at $t=T^{+}$(propagator $Q$ ). The combined propagator for this trajectory is $Q\left(A^{-1} S\right)^{n} A^{-1} R$; summing over all possible winding numbers and the direct trajectory, we obtain the total propagator 


$$
\begin{aligned}
M & =P+Q\left(\sum_{n=0}^{\infty}\left(A^{-1} S\right)^{n}\right) A^{-1} R \\
& =P+Q(A-S)^{-1} R,
\end{aligned}
$$

which agrees with the result obtained in Section 凹IIA.

\section{APPENDIX B: PARTIAL TRACE FORMALISM FOR FREE FIELDS}

In this Appendix, we derive the evolution operator for free field models in the presence of CTC's using the partial trace formulation of the self-consistent path integral.

We consider a general free theory whose Fock space is built using creation operators $a_{1}^{\dagger}, \ldots, a_{s_{1}}^{\dagger}$ and $b_{1}^{\dagger}, \ldots, b_{s_{2}}^{\dagger}$, acting on vacuum $|\mathbf{0} ; \mathbf{0}\rangle$. The $a_{i}$ and $b_{i}$ obey the CCR/CAR's. The basis elements are written!

$$
|\mathbf{m} ; \mathbf{n}\rangle=\prod_{i=1}^{s_{1}}\left(m_{i} !\right)^{-1 / 2}\left(a_{i}^{\dagger}\right)^{m_{i}} \prod_{i=1}^{s_{2}}\left(n_{i} !\right)^{-1 / 2}\left(b_{i}^{\dagger}\right)^{n_{i}}|\mathbf{0} ; \mathbf{0}\rangle,
$$

and we write $m=\sum m_{i}, n=\sum n_{i}$ etc. We will also need the alternative basis $|\mathbf{m} ; \tilde{\mathbf{n}}\rangle$ defined by

$$
|\mathbf{m} ; \tilde{\mathbf{n}}\rangle=\prod_{i=1}^{s_{1}}\left(m_{i} !\right)^{-1 / 2}\left(a_{i}^{\dagger}\right)^{m_{i}} \prod_{i=1}^{s_{2}}\left(n_{i} !\right)^{-1 / 2}\left(\left(A^{\dagger} b\right)_{i}^{\dagger}\right)^{n_{i}}|\mathbf{0} ; \mathbf{0}\rangle .
$$

Suppose the evolution $U$ on Fock space is unitary and such that

$$
\begin{aligned}
a_{i}(T) & =U^{\dagger} a_{i} U=P_{i j} a_{j}+Q_{i j} b_{j} \\
b_{i}(T) & =U^{\dagger} b_{i} U=R_{i j} a_{j}+S_{i j} b_{j},
\end{aligned}
$$

where the matrix

$$
V=\left(\begin{array}{ll}
P & Q \\
R & S
\end{array}\right)
$$

is unitary. We note that this entails that $U$ preserves the total particle number $\sum a_{i}^{\dagger} a_{i}+$ $\sum b_{i}^{\dagger} b_{i}$.

We now specialise to the bosonic case. From Section $\llbracket \mathrm{XA}$, the evolution operator $X$ has matrix elements given by

$$
\left\langle\mathbf{m} ; \mathbf{n}|X| \mathbf{m}^{\prime} ; \mathbf{n}^{\prime}\right\rangle=\mathcal{N}_{\mathbf{b}} \delta_{\mathbf{n n}^{\prime}} \sum_{\mathbf{n}^{\prime \prime}}\left\langle\mathbf{m} ; \tilde{\mathbf{n}}^{\prime \prime}|U| \mathbf{m}^{\prime} ; \mathbf{n}^{\prime \prime}\right\rangle,
$$

\footnotetext{
${ }^{7}$ We note in passing that the basis used in Eq. (2) of Ref. [1] for fermionic systems is not properly anticommuting.
} 
where $\mathcal{N}_{\mathrm{b}}$ is a normalisation constant, chosen to ensure that $\langle\mathbf{0}|X| \mathbf{0}\rangle=1$ (as it should be for any free theory). This allows us to evaluate $\mathcal{N}_{\mathrm{b}}$ explicitly, because the matrix element $\langle\mathbf{0} ; \tilde{\mathbf{n}}|U| \mathbf{0} ; \mathbf{n}\rangle$ is

$$
\langle\mathbf{0} ; \tilde{\mathbf{n}}|U| \mathbf{0} ; \mathbf{n}\rangle=\left\langle\mathbf{0} ; \mathbf{0}\left|\prod_{i} \frac{\left(A^{\dagger} b\right)_{i}^{n_{i}}}{\left(n_{i} !\right)^{1 / 2}} U\right| \mathbf{0} ; \mathbf{n}\right\rangle=\left\langle\mathbf{0} ; \mathbf{0}\left|U \prod_{i} \frac{\left(A^{\dagger} b(T)\right)_{i}^{n_{i}}}{\left(n_{i} !\right)^{1 / 2}}\right| \mathbf{0} ; \mathbf{n}\right\rangle,
$$

and is therefore equal to the coefficient of $\prod_{i} x_{i}^{n_{i}}$ in the expansion of $\prod_{i}\left(\sum_{j}\left(A^{\dagger} S\right)_{i j} x_{j}\right)^{n_{i}}$. We have used the fact that $U$ preserves the vacuum. The generating function $G\left(x_{1}, \ldots, x_{s_{2}}\right)$ for these coefficients can be found in $\S 66$ of [33], and is given by

$$
G\left(x_{1}, \ldots, x_{s_{2}}\right)=\frac{(-1)^{s_{2}}\left(x_{1} x_{2} \ldots x_{s_{2}}\right)^{-1}}{\operatorname{det}\left(A^{\dagger} S-\operatorname{diag}\left(x_{1}^{-1}, x_{2}^{-1}, \ldots, x_{s_{2}}^{-1}\right)\right)} .
$$

The sum over all $\mathbf{n}$ of these matrix elements is obtained simply by evaluating the generating function with all $x_{i}$ equal to unity. Thus we obtain

$$
\mathcal{N}_{\mathrm{b}}=\operatorname{det}\left(\mathbb{1}-A^{\dagger} S\right)
$$

Next, we claim that

$$
X^{-1} a_{i} X=M_{i j} a_{j}
$$

where $M=P+Q(A-S)^{-1} R$ is unitary. Together with the trivial evolution $X^{-1} b_{i} X=B_{i j} b_{j}$, this shows that $X$ is unitary. Moreover, this is the free evolution derived in various ways in the body of the paper.

To establish ( $\mathrm{B} 9)$, we first note that

$$
\begin{aligned}
\sum_{\mathbf{n}}\left\langle\mathbf{m} ; \tilde{\mathbf{n}}\left|U b_{i}\right| \mathbf{m}^{\prime} ; \mathbf{n}\right\rangle & =\sum_{\mathbf{n}}\left\langle\mathbf{m} ; \tilde{\mathbf{n}}\left|\left(A^{\dagger} b\right)_{i} U\right| \mathbf{m}^{\prime} ; \mathbf{n}\right\rangle \\
& =\sum_{\mathbf{n}}\left\langle\mathbf{m} ; \tilde{\mathbf{n}}\left|U A_{i j}^{\dagger}\left(R_{j k} a_{k}+S_{j k} b_{k}\right)\right| \mathbf{m}^{\prime} ; \mathbf{n}\right\rangle
\end{aligned}
$$

where the first step follows by relabelling the sum over $n_{i}$. Collecting terms in the $b_{i}$ and rearranging, we have

$$
\sum_{\mathbf{n}}\left\langle\mathbf{m} ; \tilde{\mathbf{n}}\left|U b_{i}\right| \mathbf{m}^{\prime} ; \mathbf{n}\right\rangle=\sum_{\mathbf{n}}\left\langle\mathbf{m} ; \tilde{\mathbf{n}}\left|U(A-S)_{i j}^{-1} R_{j k} a_{k}\right| \mathbf{m}^{\prime} ; \mathbf{n}\right\rangle
$$

and hence

$$
\begin{aligned}
\sum_{\mathbf{n}}\left\langle\mathbf{m} ; \tilde{\mathbf{n}}\left|a_{i} U\right| \mathbf{m}^{\prime} ; \mathbf{n}\right\rangle & =\sum_{\mathbf{n}}\left\langle\mathbf{m} ; \tilde{\mathbf{n}}\left|U\left(P_{i j} a_{j}+Q_{i j} b_{j}\right)\right| \mathbf{m}^{\prime} ; \mathbf{n}\right\rangle \\
& =\sum_{\mathbf{n}}\left\langle\mathbf{m} ; \tilde{\mathbf{n}}\left|U M_{i j} a_{j}\right| \mathbf{m}^{\prime} ; \mathbf{n}\right\rangle,
\end{aligned}
$$

where $M=P+Q(A-S)^{-1} R$. Thus we have $a_{i} X=X M_{i j} a_{j}$ as required.

In the fermionic case, we define the operator $X$ by 


$$
\left\langle\mathbf{m} ; \mathbf{n}|X| \mathbf{m}^{\prime} ; \mathbf{n}^{\prime}\right\rangle=\mathcal{N}_{\mathrm{f}} \delta_{\mathbf{n n}^{\prime}} \sum_{\mathbf{n}^{\prime \prime}}(-1)^{n^{\prime \prime}}\left\langle\mathbf{m} ; \tilde{\mathbf{n}}^{\prime \prime}|U| \mathbf{m}^{\prime} ; \mathbf{n}^{\prime \prime}\right\rangle
$$

where $\mathcal{N}_{\mathrm{f}}$ is chosen to ensure that $\langle\mathbf{0}|X| \mathbf{0}\rangle=1$. The factor of $(-1)^{n^{\prime \prime}}$ is necessary in order to obtain agreement with the canonical theory. To see this, note that the first step in (B10) is not valid in the fermionic case, due to the anticommutation relations satisfied by the $a_{i}$ and $b_{i}$ and the definition (B1). Instead, the corresponding result is

$$
\sum_{\mathbf{n}}(-1)^{n\left(m+m^{\prime}\right)}\left\langle\mathbf{m} ; \tilde{\mathbf{n}}\left|U b_{i}\right| \mathbf{m}^{\prime} ; \mathbf{n}\right\rangle=\sum_{\mathbf{n}}(-1)^{n\left(m+m^{\prime}\right)}\left\langle\mathbf{m} ; \tilde{\mathbf{n}}\left|\left(A^{\dagger} b\right)_{i} U\right| \mathbf{m}^{\prime} ; \mathbf{n}\right\rangle,
$$

in which the factors of $(-1)^{m}$ and $(-1)^{m^{\prime}}$ arise from anticommuting $b_{i}$ past the string of creation operators for $|\mathbf{m}\rangle$ and $\left|\mathbf{m}^{\prime}\right\rangle$ respectively. We may replace $(-1)^{n\left(m+m^{\prime}\right)}$ by $(-1)^{n}$ because $U$ preserves the total particle number and therefore the summands can be nonzero only when $m^{\prime}=m+1$.

Exactly analogous arguments to those for the bosonic case then show that eq. (B9) holds, and that $X$ is unitary. Thus we have obtained agreement with the canonical theory.

The constant $\mathcal{N}_{\mathrm{f}}$ is easily evaluated once it has been expressed in the form

$$
\mathcal{N}_{\mathrm{f}}^{-1}=\left\langle\mathbf{0} ; \mathbf{0}\left|\left[\bigwedge^{s_{2}}\left(\mathbb{1}-A^{\dagger} S\right) b_{s_{2}} \ldots b_{1}\right] b_{1}^{\dagger} \ldots b_{s_{2}}^{\dagger}\right| \mathbf{0} ; \mathbf{0}\right\rangle,
$$

for then one may use the exterior algebra definition of the determinant 8 to conclude that

$$
\mathcal{N}_{\mathrm{f}}=\left[\operatorname{det}\left(\mathbb{1}-A^{\dagger} S\right)\right]^{-1}
$$

To establish Eq. (B15), we write its RHS as $\mathcal{N}^{-1}$ and expand the exterior power to obtain

$$
\mathcal{N}^{-1}=\sum_{\mathbf{n}}\left\langle\mathbf{0} ; \mathbf{0}\left|(-1)^{n} c_{s_{2}}^{\left(n_{s_{2}}\right)} \ldots c_{1}^{\left(n_{1}\right)} b_{1}^{\dagger} \ldots b_{s_{2}}^{\dagger}\right| \mathbf{0} ; \mathbf{0}\right\rangle
$$

where $c_{i}^{\left(n_{i}\right)}$ is defined to be equal to $b_{i}$ if $n_{i}=0$ or $\left(A^{\dagger} S b\right)_{i}$ if $n_{i}=1$. Next, move the leftmost $c_{i}^{\left(n_{i}\right)}$ with $n_{i}=0$ rightwards using the anticommutation relations until it sits next to $b_{i}^{\dagger}$, at which point the $b_{i} b_{i}^{\dagger}$ combination may be removed by a further application of the CAR's. Repeating the process until all $c_{i}^{(0)}$ 's have been removed, one eventually finds

$$
\mathcal{N}^{-1}=\sum_{\mathbf{n}}(-1)^{n}\left\langle\mathbf{0} ; \mathbf{0}\left|\left(A^{\dagger} S b\right)_{i}^{n_{i}}\right| \mathbf{0} ; \mathbf{n}\right\rangle
$$

which is easily shown to be equal to $\sum_{\mathbf{n}}(-1)^{n}\langle\mathbf{0} ; \tilde{\mathbf{n}}|U| \mathbf{0} ; \mathbf{n}\rangle=\mathcal{N}_{\mathrm{f}}^{-1}$, thus verifying our claim.

\footnotetext{
${ }^{8}$ Recall that if $Q$ is an endomorphism of a vector space with anticommuting basis $b_{1}, \ldots, b_{N}$, then $\left(\bigwedge^{N} Q\right) b_{N} \ldots b_{1}=(Q b)_{N} \ldots(Q b)_{1}=(\operatorname{det} Q) b_{N} \ldots b_{1}$.
} 


\section{APPENDIX C: VIOLATION OF CCR/CAR'S IN THE 3-POINT MODEL}

We present here the details of the calculation leading to Eqs. (5.21), (5.22) and (5.31) and the statements made at the end of Sect. VIII. We consider the 1-parameter family of operator orderings labelled by $\alpha \in[0,1]$ discussed in Sect. VIII for which

$$
\Psi(t)=e^{-i \lambda T \alpha \Psi(0)^{\dagger} \Psi(0)}\left(e^{-i W T} \Psi(0)\right) e^{-i \lambda T(1-\alpha) \Psi(0)^{\dagger} \Psi(0)},
$$

and consider Bose and Fermi statistics simultaneously, seeking solutions of the form $b=$ $f\left(d^{\dagger} d, c^{\dagger} c\right) c$ where

$$
c=\frac{R_{1} a_{1}+R_{2} a_{2}}{\|R\|} \quad \text { and } \quad d=\frac{\bar{R}_{2} a_{1}-\bar{R}_{1} a_{2}}{\|R\|}
$$

obey the same commutation relations as $a_{1}$ and $a_{2}$.

Applying $b$ to elements of form $\left(d^{\dagger}\right)^{m} c^{\dagger}|0\rangle$, we obtain the consistency requirement

$$
f(m, 0)=(\|R\|+S f(m, 0)) e^{-i \lambda T\left[m+(1-\alpha)\left(1+|f(m, 0)|^{2}\right)\right]},
$$

and applying $b$ to elements of form $\left(d^{\dagger}\right)^{m}\left(c^{\dagger}\right)^{n+2}|0\rangle$ for $m, n \geq 0$, we obtain the recursion relation

$$
f(m, n+1)=(\|R\|+S f(m, n+1)) e^{-i \lambda T\left[m+\alpha(n+1)\left(1+|f(m, n)|^{2}\right)+(1-\alpha)(n+2)\left(1+|f(m, n+1)|^{2}\right)\right]} .
$$

We compute the quantity

$$
J=\left\langle 0\left|\left(a_{2}(T) a_{1}(T) \mp a_{1}(T) a_{2}(T)\right) d^{\dagger} c^{\dagger}\right| 0\right\rangle
$$

for Bose (-) and Fermi (+) statistics and also

$$
K=\left\langle 0\left|a_{1}(T)^{2} d^{\dagger} c^{\dagger}\right| 0\right\rangle
$$

for Fermi statistics. Note that $d^{\dagger} c^{\dagger}|0\rangle$ is an element of $\mathfrak{F}_{2}$; in the fermionic case it is $a_{1}^{\dagger} a_{2}^{\dagger}|0\rangle$.

First note that

$$
\left\langle 0\left|a_{i}(T) a_{j}(T) d^{\dagger} c^{\dagger}\right| 0\right\rangle=e^{-i \omega}\left\langle 0\left|\left(P_{i k} a_{k}+Q_{i} b\right) e^{-i \lambda T c^{\dagger}\left|f\left(d^{\dagger} d, c^{\dagger} c\right)\right|^{2} c}\left(P_{j l} a_{l}+Q_{j} b\right) d^{\dagger} c^{\dagger}\right| 0\right\rangle,
$$

where $\omega=\lambda T\left[(1-\alpha)\left(2+|f(1,0)|^{2}\right)+1\right]$. We have

$$
\begin{aligned}
P_{1 i} a_{i} & =\frac{P_{1 i} \bar{R}_{i}}{\|R\|} c+\frac{P_{11} R_{2}-P_{12} R_{1}}{\|R\|} d \\
& =-\frac{Q_{1} \bar{S}}{\|R\|} c-\frac{\bar{Q}_{2} \operatorname{det} P}{\bar{S}\|R\|} d
\end{aligned}
$$

where we have used the identities

$$
P_{i j} \bar{R}_{j}=-Q_{i} \bar{S}
$$


and

$$
P_{11} R_{2}-P_{12} R_{1}=-\frac{\bar{Q}_{2} \operatorname{det} P}{\bar{S}}
$$

which follow from the unitarity of $e^{-i W T}$. The second of these is derived by noting that $\bar{S} R_{j}=-\bar{Q}_{i} P_{i j}$. Similarly, we have

$$
P_{2 i} a_{i}=-\frac{Q_{2} \bar{S}}{\|R\|} c+\frac{\bar{Q}_{1} \operatorname{det} P}{\bar{S}\|R\|} d
$$

in which we have used $P_{21} R_{2}-P_{22} R_{1}=\overline{Q_{1}} \operatorname{det} P / \bar{S}$.

We can now compute

$$
\begin{aligned}
\left\langle 0\left|a_{2}(T) a_{1}(T) d^{\dagger} c^{\dagger}\right| 0\right\rangle= & e^{-i \omega} \frac{\operatorname{det} P}{\bar{S}\|R\|}\left\{ \pm\left|Q_{1}\right|^{2}\left(f(1,0)-\frac{\bar{S}}{\|R\|}\right)-\right. \\
& \left.\left|Q_{2}\right|^{2}\left(f(0,0)-\frac{\bar{S}}{\|R\|}\right) e^{-i \lambda T|f(0,0)|^{2}}\right\},
\end{aligned}
$$

where the \pm is + for Bose and - for Fermi statistics. To obtain $J$, we interchange the suffices 1 and 2 and add for Bose and subtract for Fermi. The sign reversal occurs because $d^{\dagger} c^{\dagger}|0\rangle$ flips sign under the interchange. The final result is then

$$
J=e^{-i \omega} \operatorname{det} P\left( \pm\left|Q_{1}\right|^{2}+\left|Q_{2}\right|^{2}\right)\left\{\frac{f(1,0)-f(0,0) e^{-i \lambda T|f(0,0)|^{2}}}{\bar{S}\|R\|}+\frac{e^{-i \lambda T|f(0,0)|^{2}}-1}{\|R\|^{2}}\right\} .
$$

The matrix element $K$ may be computed for Fermi statistics as

$$
K=e^{-i \omega} Q_{1} \bar{Q}_{2} \operatorname{det} P\left\{\frac{f(1,0)-f(0,0) e^{-i \lambda T|f(0,0)|^{2}}}{\bar{S}\|R\|}+\frac{e^{-i \lambda T|f(0,0)|^{2}}-1}{\|R\|^{2}}\right\} .
$$

With the particular operator ordering used in Section $\nabla \mathrm{V}(\alpha=1)$, we have $f(0,0)=$ $\|R\|(1-S)^{-1}$ and $f(1,0)=\|R\|\left(e^{i \lambda T}-S\right)^{-1}$. Substituting these values into Eqs. (C13) and ([14) and using the fact that $\|R\|^{2}=1-|S|^{2}$, we obtain Eqs. (5.21), (5.22) and (5.31) respectively.

Finally, one should also check that the expression enclosed within braces in Eqs. (C13) and (C14) does not vanish. For $\lambda T \ll 1$, one may prove this by perturbing about the free solution to obtain $f(0,0)$ and $f(1,0)$ to second order in $\lambda T$ if $S \notin \mathbb{R}$. If $S$ is real, one needs to go to third order. 


\section{REFERENCES}

[1] H.D. Politzer, Phys. Rev. D49 3981 (1994)

[2] J.L. Friedman and M.S. Morris, Phys. Rev. Lett. 66401 (1991)

[3] J.L. Friedman and M.S. Morris, Existence and Uniqueness Theorems for Massless Fields on a Class of Spacetimes with Closed Timelike Curves, Preprint WISC-MILW-94-TH-22, gr-qc/9411033

[4] J. Friedman, M.S. Morris, I.D. Novikov, F. Echeverria, G. Klinkhammer, K.S. Thorne and U. Yurtsever, Phys. Rev. D42 1915 (1990)

[5] H.D. Politzer, Phys. Rev. D46 4470 (1992)

[6] D.S. Goldwirth, M.J. Perry, T. Piran and K.S. Thorne, Phys. Rev. D49 3951 (1994)

[7] F. Echeverria, G. Klinkhammer and K.S. Thorne, Phys. Rev. D44 1077 (1991)

[8] I.D. Novikov, Phys. Rev. D45 1989 (1992)

[9] S.K. Rama and S. Sen, Inconsistent Physics in the Presence of Time Machines Preprint MRI-PHY/14/94, TCD-7-94, hep-th/9410031

[10] D. Deutsch, Phys. Rev. D44 3197 (1991)

[11] J.L. Friedman, N.J. Papastamatiou and J.Z. Simon, Phys. Rev. D46 4442 (1992)

[12] D.G. Boulware, Phys. Rev. D46 4421 (1992)

[13] J.L. Friedman, N.J. Papastamatiou and J.Z. Simon, Phys. Rev. D46 4456 (1992)

[14] T. Jacobson, in Conceptual Problems of Quantum Gravity, ed. A. Ashtekar and J. Stachel, (Birkhäuser, Boston (1991))

[15] J.B. Hartle, Phys. Rev. D49 6543 (1994)

[16] A. Anderson, Phys. Rev. D51 5707 (1995)

[17] C.J. Fewster and C.G. Wells, Phys. Rev. D52 5773 (1995)

[18] S.W. Hawking, Phys. Rev. D52 5681 (1995)

[19] M.J. Cassidy, Phys. Rev. D52 5678 (1995)

[20] P.A.M. Dirac, Lectures on Quantum Mechanics (Yeshiva University, New York, 1964)

[21] C.J. Fewster and C.G. Wells, In preparation

[22] U. Yurtsever, J. Math. Phys. 313064 (1990)

[23] K. Deimling, Nonlinear Functional Analysis, (Springer-Verlag, Berlin, 1985)

[24] V.I. Arnol'd, Mathematical Methods of Classical Mechanics, 2nd edition, (Springer Verlag, New York, 1989)

[25] M. Reed and B. Simon, Methods of Modern Mathematical Physics Vol I: Functional Analysis (Academic Press, New York, 1972)

[26] F. Rellich, Nachr. d. Akad. d. Wiss. in Göttingen, Math-Phys. Kl. 107 (1946)

[27] N. Dunford and J. Schwartz, Linear Operators, Part I: General Theory (Wiley (Interscience), New York, 1958)

[28] B. Simon, in Mathematics of Contemporary Physics, ed. R.F. Streater, (Academic Press, London, 1972)

[29] W. Arveson, Acta Math. 123141 (1969)

[30] G. Klinkhammer and K.S. Thorne, Unpublished

[31] L.D. Faddeev and A.A. Slavnov, Gauge Fields: Introduction to Quantum Theory (Benjamin/Cummings, Reading M.A., 1980)

[32] M. Mnatsakanova, G. Morchio, F. Strocchi and Yu. Vernov, Irreducible Representations of the Heisenberg Algebra in Krein Spaces, Preprint IFUP-TH 70/95

[33] P.A. MacMahon, Combinatory Analysis (Chelsea Publishing, New York, 1960) 


\section{FIGURES}

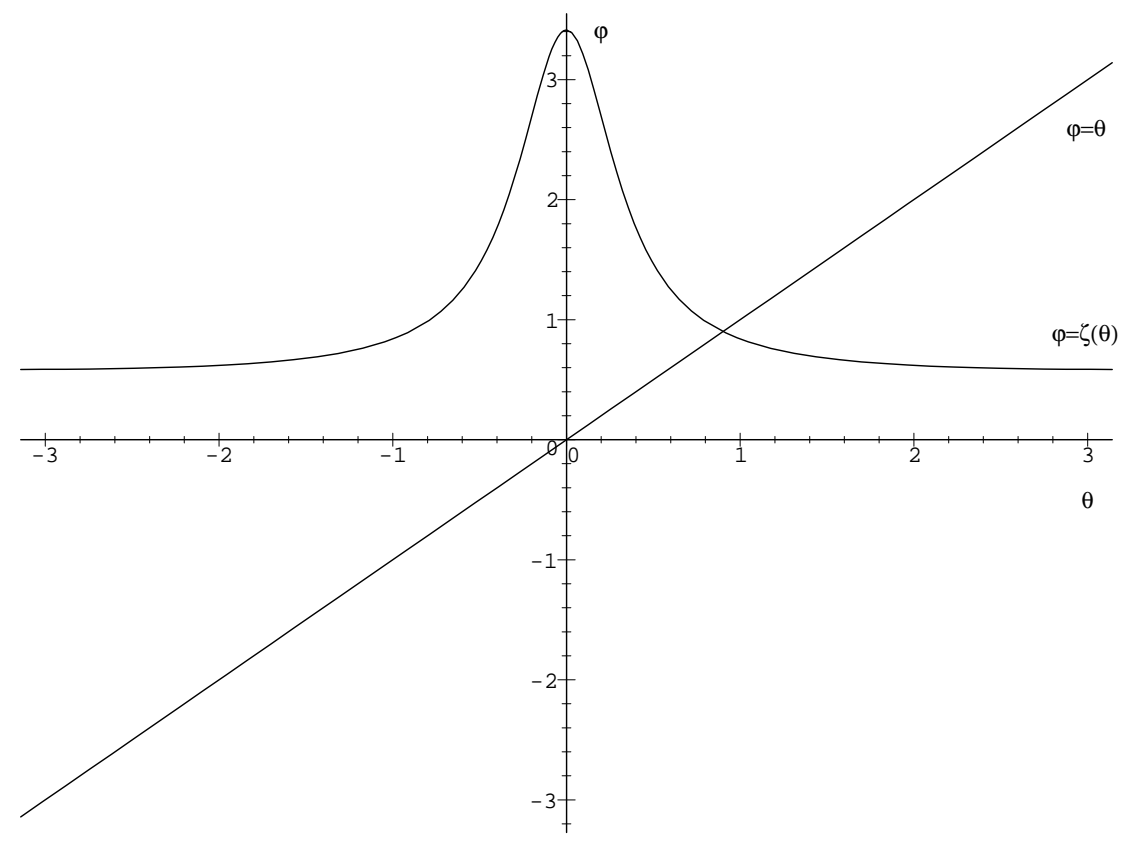

FIG. 1. Graphical solution of Eq. (3.14) for $\mu=0.5$ showing that there is a unique solution of the classical solution for this coupling strength. 


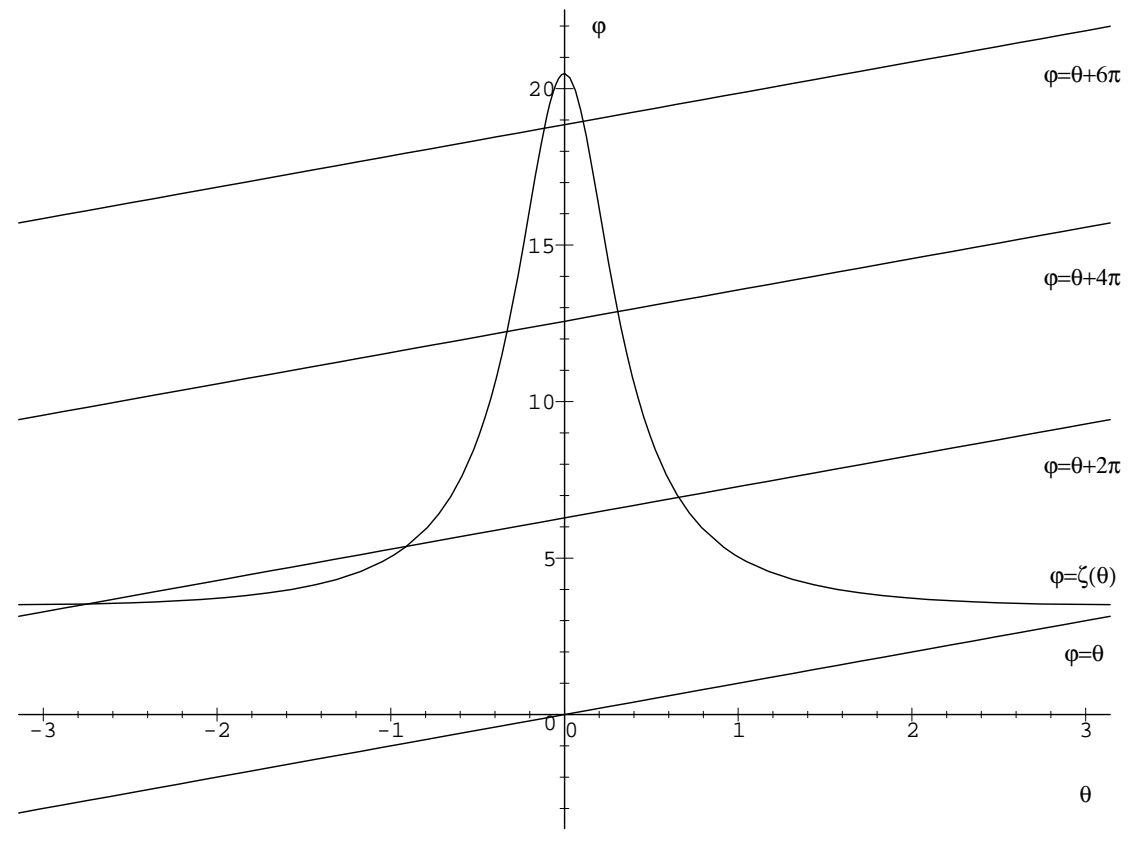

FIG. 2. Graphical solution of Eq. (3.14) for $\mu=3.0$ showing that there are 7 solutions for this coupling strength. 


\section{TABLES}

TABLE I. Table of $\theta_{n}$ defined by Eq. (7.6) for different values of the coupling strength $\mu$. The final row shows the value of $\theta$ corresponding to the unique classical limit picked out by the quantum theory.

\begin{tabular}{rrrrr}
\hline \hline$n$ & & & & \\
& & & \\
& $\mu=0.5$ & $\mu=3.0$ & $\mu=7.0$ & $\mu=13.0$ \\
\hline 500 & 0.9020 & -2.7496 & 2.2226 & 2.7558 \\
1000 & 0.9018 & -2.7492 & 2.2124 & 2.7527 \\
1500 & 0.9017 & -2.7490 & 2.2115 & 2.7516 \\
2000 & 0.9016 & -2.7489 & 2.2112 & 2.7510 \\
2500 & 0.9016 & -2.7489 & 2.2109 & 2.7507 \\
\hline Classical solution & 0.9015 & -2.7487 & 2.2101 & 2.7494 \\
\hline \hline
\end{tabular}

